\title{
Path-components of Morse mappings spaces of surfaces
}

\author{
Sergey Maksymenko*
}

\begin{abstract}
Let $M$ be a connected compact surface, $P$ be either $\mathbb{R}^{1}$ or $S^{1}$, and $\mathcal{F}(M, P)$ be the space of Morse mappings $M \rightarrow P$ with compact-open topology. The classification of pathcomponents of $\mathcal{F}(M, P)$ was independently obtained by S. V. Matveev and V. V. Sharko for the case $P=\mathbb{R}^{1}$, and by the author for orientable surfaces and $P=S^{1}$. In this paper we give a new independent and unified proof of this classification for all compact surfaces in the case $P=\mathbb{R}$, and for orientable surfaces in the case $P=S^{1}$. We also extend the author's initial proof to non-orientable surfaces.
\end{abstract}

Mathematics Subject Classification (2000). 37E30, 58B05.

Keywords. Surface, Morse mapping, mapping class group, Torelli group.

\section{Introduction}

Let $M$ be a smooth $\left(C^{\infty}\right)$ connected compact surface with boundary $\partial M$ (possibly empty) and let $P$ be a one-dimensional manifold, i.e. either the real line $\mathbb{R}^{1}$ or the circle $S^{1}$. Consider the subspace $\mathcal{F}(M, P)$ of $C^{\infty}(M, P)$ consisting of Morse mappings $M \rightarrow P$. It is well-known that $\mathcal{F}(M, P)$ is an everywhere dense open subset of $C^{\infty}(M, P)$ in the compact-open topology of $C^{\infty}(M, P)$. The homotopy type of this space is of great importance in differential topology and dynamical systems, see e.g. [H], [I], [HT], [HH], [KE], [SV1], [M], [IS].

Recently, S. V. Matveev and V. V. Sharko [SV1] have obtained a full description of path-components of the space $\mathcal{F}\left(M, \mathbb{R}^{1}\right)$. Matveev's proof is included and generalized in the paper $[\mathrm{KE}]$ of E. Kudryavtseva to numerated Morse functions. Their proofs were independent and based on different ideas. The classification of pathcomponents of $\mathcal{F}\left(M, S^{1}\right)$ for orientable surfaces was given in the author's Ph.D. thesis, see $[\mathrm{M}]$.

These results (which we will refer to as Main Theorem) can be summarized as follows: two Morse mappings $f, g: M \rightarrow P$ belong to same path-component of $\mathcal{F}(M, P)$ if and only if they are homotopic as continuous maps and have the same

\footnotetext{
*The author is partially supported by the grant of Government Fond of Fundamental Researches no. 1.7/132
} 
number of critical points at each index and the same sets of positive and negative boundary components (in the sense described below.)

In this paper we give a unified and independent proof of this theorem for all compact surfaces in the case $P=\mathbb{R}$. The case of Morse mappings $M \rightarrow S^{1}$ requires information on the subgroup of the mapping class group of $M$ preserving a given element in the cohomology group $H^{1}(M, \mathbb{Z})$. We also find the generators of this group for orientable surfaces and extend the presented method to Morse mappings from orientable surfaces into $S^{1}$.

In fact, the proof given in $[\mathrm{M}]$ for this case almost literally extends to nonorientable surfaces as well. Since $[\mathrm{M}]$ was never published in English, we give this proof for all surfaces in the Appendix. Thus the Main Theorem is proved here for all cases of $M$ and $P$.

Our approach has a relation to the paper [HT] of A. Hatcher and W. Thurston, who used deformations of Morse functions to construct a representation for the mapping class group of a surface. In constrast to this approach, we exploit generators of this group to find a deformation between Morse mappings in $\mathcal{F}(M, P)$. The principal observation is that "elementary diffeomorphisms" like Dehn twists, boundary and crosscap slides generating mapping class groups of surfaces preserve certain Morse functions.

\section{Preliminaries}

Let $M$ be a compact surface. A surface obtained by shrinking every connected component of $M$ to a point will be denoted by $\widehat{M}$. Thus $\widehat{M}$ is closed and is homeomorphic with a connected sum of the form either $S^{2} \#_{i=1}^{g} T^{2}$ (orientable case, $g \geq 0$ ) or $\#_{i=1}^{g} \mathbb{P}^{2}$ (non-orientable case, $g \geq 1$ ). In each of the cases the number $g$ is called the germ of $M$. All homology and cohomology groups will be taken with integer coefficients. The term simple closed curve will be abbreviated to SCC. The circle $S^{1}$ will be regarded as the subset $\{z \in \mathbb{C}:|z|=1\}$ of the complex plane $\mathbb{C}$. For a topological space $X$ let \#[X] denote the number of its connected components.

2.1. Morse mappings. Let us fix, once and for all, an orientation of $P$. Consider a smooth mapping $f: M \rightarrow P$. A point $z \in M$ is critical for $f$ if $d f(z)=0$. A critical point $z$ of $f$ is non-degenerate if the Hessian of $f$ at $z$ is non-degenerate. Suppose that $z$ is a non-degenerate critical point of $f$. Then by Morse lemma there are embeddings $p: \mathbb{R}^{2} \rightarrow M$ and $q: \mathbb{R}^{1} \rightarrow P$ onto open neighborhoods of $z$ and $f(z)$ respectively such that $p(0,0)=z, q(0)=f(z), q$ preserves orientation, and $q^{-1} \circ f \circ p(x, y)= \pm x^{2} \pm y^{2}$. The number of minuses in this representation does not depend on a particular choice of such embeddings and is called the index of a critical point $z$.

A $C^{\infty}$-mapping $f: M \rightarrow P$ is Morse if the following conditions hold: 
(1) all critical points of $f$ are non-degenerate and belong to the interior of $M$;

(2) $f$ is constant at each boundary component of $M$ while its values on different components may differ each from other.

The subspace of $C^{\infty}(M, P)$ consisting of Morse mappings will be denoted by $\mathcal{F}(M, P)$. We endow $C^{\infty}(M, P)$ with the compact-open topology. Then this topology induces some topology on $\mathcal{F}(M, P)$.

2.2. $\Sigma$-homotopies. Let $f, g \in \mathcal{F}(M, P)$ be two Morse mappings and $\phi:[0,1] \rightarrow$ $C^{\infty}(M, P)$ be a path between them in the space of Morse mappings. Thus $\phi$ is continuous, $\phi(0)=f, \phi(1)=g$ and $\phi(t)$ is Morse for all $t \in[0,1]$. Then $\phi$ yields a continuous mapping (homotopy) $F: M \times I \rightarrow P$ such that $F_{0}=f, F_{1}=g$, and $F_{t}$ is Morse for all $t \in I$. In particular, $F$ is $C^{\infty}$ in $x \in M$ but may be just continuous in $t \in[0,1]$. Conversely, every such mapping $F$ gives rise to a path between $f$ and $g$ in $\mathcal{F}(M, P)$.

We will call the mapping $F$ a $\Sigma$-homotopy or $\Sigma$-deformation between $f$ and $g$ and write $f \stackrel{F_{t}}{\sim} g$. The term $f \stackrel{\Sigma}{\sim} g$ will also be used to indicate that $f$ and $g$ are $\Sigma$-homotopic.

Remark 2.1. In [SV1], [KE], $\Sigma$-homotopies are called isotopies of Morse functions. We will use another term in order to avoid confusions with isotopies of diffeomorphisms.

2.3. Invariants of $\Sigma$-homotopies. Let $f \in \mathcal{F}(M, P)$. The objects (i) homotopy class, (ii) number of critical points in each index, and (iii) positive and negative boundary components are invariant under $\Sigma$-homotopies of $f$.

2.3.1. Homotopy class. First suppose that $P=S^{1}$. Let $\xi \in H^{1}\left(S^{1}\right)$ be a generator defining the chosen orientation of $S^{1}$. If $f: M \rightarrow S^{1}$ is a continuous mapping, then the correspondence $f \mapsto f^{*}(\xi) \in H^{1}(M)$ yields a bijection between the set of homotopy classes of mappings $\left[M, S^{1}\right]$ and the cohomology group $H^{1}(M)$. Since by our definition Morse mappings are constant at the connected components of $\partial M$, it follows that the set of homotopy classes of Morse mappings $M \rightarrow S^{1}$ is bijective to the group $H^{1}(\widehat{M})$ for the corresponding closed surface $\widehat{M}$.

Let $g$ be a genus of $M$. A simple calculation shows that $H^{1}(\widehat{M})$ is isomorphic with $\mathbb{Z}^{r}$, where $r$ is either $2 g$ or $g-1$ provided $M$ is orientable or not. Let us fix a basis for $H^{1}(\widehat{M})$. Then the homotopy class of $f$ is an integer vector

$$
\left(q_{1}, \ldots, q_{r}\right)=f(\xi) \in H^{1}(\widehat{M})=\mathbb{Z}^{r} .
$$

For $P=\mathbb{R}$ we will assume that $\left(q_{1}, \ldots, q_{r}\right)=(0, \ldots, 0)$. 
2.3.2. Number of critical points in each index. Denote by $c_{i}(f)=c_{i}(i=0,1,2)$ the number of critical points of $f$ of index $i$. Then by Morse equalities we have

$$
c_{0}(f)+c_{1}(f)-c_{2}(f)=\chi(M) .
$$

2.3.3. Positive and negative components of $\partial M$. Let $V$ be a component of $\partial M$, $z \in V$ and let $\xi \in T M_{z}$ be a tangent vector at $z$ directed outward $M$. Denote by $\varepsilon_{f}(V)$ the sign of the value $d f(z) \xi$. Since $f$ has no critical points on $V$, we see that $\varepsilon_{f}(V)= \pm 1$ and does not depend on a particular choice of a point $z \in V$ and a vector $\xi \in T M_{z}$ as above. Thus we get a function $\varepsilon_{f}: \pi_{0} \partial M \rightarrow\{ \pm 1\}$. We may also think of $\varepsilon_{f}$ as an element of $\{ \pm 1\}^{b}$, where $b$ is the number of connected components of $\partial M$.

We will call $V$ either $f$-positive or $f$-negative in accordance with $\varepsilon_{f}(V)$. Let $\partial_{+} M$ (resp. $\partial_{-} M$ ) be the union of $f$-positive (resp. $f$-negative) boundary components of $\partial M$, and let $b_{+}$(resp. $b_{-}$) denote the numbers of these components.

The following collection of numbers

$$
K(f)=\left\{q_{1}, \ldots, q_{r}, c_{0}, c_{1}, c_{2}, \varepsilon_{f}\right\}
$$

will be called the critical type of a Morse mapping $f$. It can be regarded as a point in $\mathbb{Z}^{r} \times \mathbb{N}_{0}^{3} \times\{ \pm 1\}^{b}$ belonging to the "hyperplane" defined by Eq. (2.1), where $\mathbb{N}_{0}=\mathbb{N} \cup\{0\}$. If we choose another orientation of $P$, then $c_{0}(f)$ exchanges with $c_{2}(f), c_{1}(f)$ remains unchanged, $\varepsilon_{f}$ and every $q_{i}$ change their signs.

Our aim is to give a new proof of the following theorem:

Main Theorem (Matveev [KE], Sharko [SV1], Maksymenko [M]). Two Morse mappings $f, g: M \rightarrow P$ belong to the same path-component of $\mathcal{F}(M, P)$ if and only if $K(f)=K(g)$, i.e. they are homotopic, have the same number of critical points in each index, and the same sets of positive and negative components of $\partial M$.

The necessity is obvious therefore we confine ourself to the sufficiency. Let us briefly review the known proofs of this theorem. First consider the case $P=\mathbb{R}^{1}$. Let $f$ and $g$ be two Morse functions with equal critical types. In both proofs [KE], [SV1] the problem was reduced to minimal Morse functions with no critical points of index 0 and 2.

Let $F$ be a gradient-like vector field for a minimal Morse function $f$. Consider a union of $f$-negative boundary components of $M$ with trajectories of $F$ that finish at critical points of $f$. This set is called a spine of $M$. Matveev (see Kudryavtseva [KE]) notes that the space of Morse functions with isotopic spines is path-connected. He further suggested elementary transformations of spines which induce $\Sigma$-homotopies of Morse function and showed that any two spines can be connected by a finite sequence of these transformations. 
Sharko [SV1] reduced the problem to minimal Morse functions on a surface $M$ with only one positive and only one negative boundary component.Such a surface can be regarded as a "framed" chords diagram in which the union of all chords and a negative boundary component constitute the spine of $M$. Notice that $\pi_{1} M$ is free. Choose a basis of this group. Then the edges of any other chords diagram in $M$ can be written down as words in the terms of a given basis. These words also form the basis of $\pi_{1} M$ and determine chord diagrams up to equivalence. Moreover, by the well-known Nielsen theorem any two bases of a finitely generated free group are related by a finite sequence of Nielsen transformations. Sharko proved that Nielsen transformations yield $\Sigma$-homotopies between corresponding Morse functions, and that Morse functions with equivalent diagrams are $\Sigma$-homotopic.

The extension of the proof of [M] for $P=S^{1}$ and all surfaces is given in the Appendix.

2.4. Plan of the present proof. First the problem will be reduced to the case when $g=f \circ h$, where $h$ is a diffeomorphism of $M$ and $f$ is of a special "canonical" form. It is convenient to say that a diffeomorphism $h$ is $f$-admissible if $f \stackrel{\Sigma}{\sim} f \circ h$. Using a special type of $f$, we will choose a system of generators for $\mathcal{M}(M)$ and show that if $P=\mathbb{R}$, then all of them are $f$-admissible. This will prove the Main Theorem for this case.

For the case $P=S^{1}, M$ is orientable, and $f$ is not null-homotopic we shall see that one of the generators chosen above is not $f$-admissible. Nevertheless, since $f$ and $f \circ h$ are homotopic, it will be possible to reduce the problem to the case when $h$ acts trivially on the homology group $H_{1}(M, \partial M)$, i.e. $h$ belongs to the Torelli group of $M$. Generators of this group are known from [P], [J], [MG]. This information will allow us to show that $f \stackrel{\Sigma}{\sim} f \circ h$.

2.5. Structure of the paper. In Section 3 we prove some technical results concerning Morse mappings to the circle. In Section 4 we recall the definition of the Kronrod-Reeb graph of a Morse mapping and define "canonical" Morse mappings. In Section 5 we reduce the Main Theorem to the case when $f$ is canonical and $g$ differs from $f$ by a diffeomorphism. This was done by Kudryavtseva in [KE] for Morse functions. We consider the case $P=S^{1}$. In Section 6 we show that elementary diffeomorphisms generating mapping class groups $\mathcal{M}(M)$ of $M$ (Dehn twists, boundary and crosscap slides) preserve certain Morse functions. In Section 7 we recall the generators of mapping class groups for surfaces with boundary. Every canonical Morse mapping gives a "canonical" set of such generators whose admissibility (or nonadmissibility) for this map is almost obvious. We also complete the Main Theorem for $P=\mathbb{R}$ (statement (i) of Lemma 7.3).

In Section 8 we give the plan of the proof of the Main Theorem for the case $M$ is orientable and $P=S^{1}$. For this in Section 9 we consider the stabilizers of elements 
of $\mathbb{Z}^{2 g}$ with respect to the action of the symplectic groups $\mathrm{Sp}_{2 g}(\mathbb{Z})$, in Section 10 we study minimal Morse functions. Section 11 includes one technical lemma. Finally, in Sections 12-14 we complete the proof.

\section{Cutting $M$ along a regular level-set of $f$}

We prove here two lemmas which will be used in the proof of Proposition 5.2.

Let $c$ be a regular value of a Morse mapping $f: M \rightarrow S^{1}$. Then $f^{-1}(c)$ is a disjoint union of SCCs on $M$. Suppose that $f^{-1}(c) \cap \partial M=\varnothing$. We cut $M$ along $f^{-1}(c)$ and denote the new surface by $\widetilde{M}=\widetilde{M}(f, c)$. Similarly, we cut $S^{1}$ at $f(c)$ and obtain [0,1]. Let $p: \widetilde{M} \rightarrow M$ and $q:[0,1] \rightarrow S^{1}$ be the corresponding factor-maps, where $q(t)=e^{2 \pi i t}, t \in[0,1]$. Then there exists a Morse function $\widetilde{f}: \widetilde{M} \rightarrow[0,1]$ such that the following diagram is commutative:

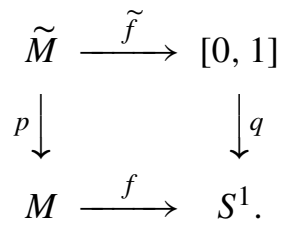

Thus

$$
f(x)=\exp \left(2 \pi i \tilde{f}\left(p^{-1}(x)\right)\right) \quad \text { for all } x \in M .
$$

Denote $B_{0}=\tilde{f}^{-1}(0), B_{1}=\tilde{f}^{-1}(1)$, and $B=B_{0} \cup B_{1}$. Then there is a natural correspondence between $\Sigma$-homotopies $\widetilde{f}_{t}$ of $\tilde{f}$ with respect to some neighborhood of $B$ and $\Sigma$-homotopies $f_{t}$ of $f$ with respect to some neighborhood of $\gamma$. The corresponding maps $\widetilde{f}_{t}$ and $f_{t}$ are related by the commutative diagram (3.1).

Since $M$ is connected, it follows that every connected component $X$ of $\tilde{M}$ intersects $B$ non trivially. However, it is possible that $X \cap B_{i}=\varnothing$ for some $i=0,1$. Thus the components of $\widetilde{M}$ can be divided into the following mutually disjoint sets

$$
Q_{0}=Q_{0}(f, c), \quad Q_{0}^{1}=Q_{0}^{1}(f, c), \quad Q^{1}=Q^{1}(f, c)
$$

consiting of those components that (respectively) have non-empty intersections only with $B_{0}$, with both sets $B_{1}$ and $B_{0}$, and with $B_{1}$ only.

It follows that for every connected component $X$ of $Q_{0}^{1}(f, c)$ and $t \in[0,1]$ we have $X \cap f^{-1}(t) \neq \varnothing$.

Lemma 3.1. (1) Let $V$ be an $\tilde{f}$-positive (resp. $\tilde{f}$-negative) component of $\partial \tilde{M}$ and $v=\tilde{f}(V)$. Then for every $w>v($ resp. $w<v)$ there exists a $\Sigma$-homotopy $\widetilde{f}_{t}$ changing $\tilde{f}$ only in an arbitrary small neighborhood of $V$ and such that $\widetilde{f}_{1}(V)=w$, see Figure 3.1a). 
(2) Let $X$ be a connected component of $\tilde{M}$. For every $w \in(0,1)$ there exists a $\Sigma$-homotopy $\widetilde{f}_{t}: \tilde{M} \rightarrow[0,1]$ such that $\widetilde{f}_{0}=\tilde{f}, \widetilde{f}_{t}=\tilde{f}$ on $(\tilde{M} \backslash X) \cup B$, and $\tilde{f}_{1}^{-1}\left(\frac{1}{2}\right) \cap X=\widetilde{f}^{-1}(w) \cap X$, see Figure 3.1b).

(3) Let $X$ be a connected component of $\tilde{M}$. Then there exists a $\Sigma$-homotopy $\widetilde{f_{t}}: \widetilde{M} \rightarrow[0,1]$ with $\widetilde{f_{0}}=\widetilde{f}$ and $\widetilde{f_{t}}=\widetilde{f}$ on $(\widetilde{M} \backslash X) \cup B$, such that $\widetilde{f}_{1}^{-1}\left(\frac{1}{2}\right) \cap X=\varnothing$, whenever $X \subset Q_{0} \cup Q^{1}$, and $\#\left[\tilde{f}_{1}^{-1}\left(\frac{1}{2}\right) \cap X\right]=1$, whenever $X \subset Q_{0}^{1}$.

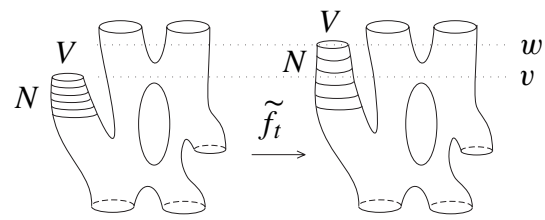

a)

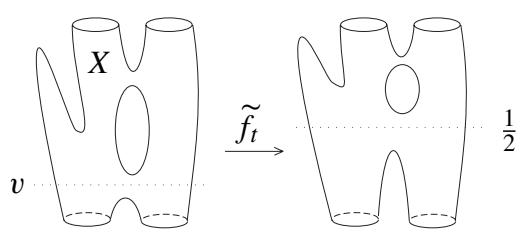

b)

Figure 3.1

Proof. (1) Suppose that $V$ is an $\tilde{f}$-positive component of $\partial \tilde{M}$. By definition, $\tilde{f}$ has no critical points on $V$. Then there exist an $\varepsilon>0$, a neighborhood $N$ of $V$, and a diffeomorphism $h: S^{1} \times(v-2 \varepsilon, v] \rightarrow N$ such that $h\left(S^{1} \times\{v\}\right)=V$ and $\tilde{f} \circ h(x, t)=t$ for $(x, t) \in S^{1} \times(v-2 \varepsilon, v]$.

Let $H_{t}$ be an isotopy of $\mathbb{R}$ fixed on $(-\infty, v-\varepsilon]$ and such that $H_{1}(v)=w$. Then the $\Sigma$-homotopy $\widetilde{f}_{t}$ defined by the formulas $\widetilde{f}_{t}(x)=\widetilde{f}(x)$ for $x \in M \backslash N$ and $\widetilde{f}_{t}(x)=H_{t} \circ \widetilde{f}(x)$ for $x \in N$ satisfies the statement (1) of the lemma. The proof for $\widetilde{f}$-negative components is similar.

(2) Notice that, for any $v \in(0,1)$, there exists an isotopy $H_{t}$ of $\mathbb{R}^{1}$ fixed near 0 and 1 and such that $H_{1}(s)=\frac{1}{2}$. Then the $\Sigma$-homotopy $\widetilde{f_{t}}: \widetilde{M} \rightarrow[0,1]$ defined by the formulas $\tilde{f}_{t}=H_{t} \circ \tilde{f}$ on $X$ and $\tilde{f_{t}}=\tilde{f}$ on $\tilde{M} \backslash X$ satisfies the statement (2) of the lemma.

(3) It follows from the definition that for every connected component $X$ of $Q_{0} \cup Q^{1}$ there exists a number $v \in(0,1)$ such that $\tilde{f}^{-1}(v) \cap X=\varnothing$. Therefore, if $X \subset$ $Q_{0} \cup Q^{1}$, then our statement follows from (2).

Let $X \subset Q_{0}^{1}$. If for some $i=0,1$ the intersection $X \cap B_{i}$ is connected, then for every $t$ in some neighborhood of $i$ we have that $X \cap \tilde{f}^{-1}(t)$ is connected. By (1) of the lemma we can choose $t=\frac{1}{2}$.

Suppose now that the intersections $X \cap B_{i}, i=0,1$ are not connected. By (1) and (2) we assume that

$$
0<\tilde{f}\left(p^{-1}\left(\partial_{-} M\right) \cap X\right)<\frac{1}{4}<\tilde{f}\left(\Sigma_{\tilde{f}} \cap X\right)<\frac{1}{2}<\tilde{f}\left(p^{-1}\left(\partial_{+} M\right) \cap X\right)<1,
$$


where $\Sigma_{\tilde{f}}$ is the set of critical points of $\tilde{f}$. Thus all critical values of $\left.\widetilde{f}\right|_{X}$ belong to $\left(\frac{1}{4}, \frac{1}{2}\right)$; the values on $\tilde{f}$-negative boundary components of $X$ except for $\tilde{f}\left(X \cap B_{0}\right)=0$ are in $\left(0, \frac{1}{4}\right)$; and the values on $\tilde{f}$-positive boundary components of $X$ except for $\tilde{f}\left(X \cap B_{1}\right)=1$ are in $\left(\frac{1}{2}, 1\right)$. In particular, $\frac{1}{2}$ is a regular value of $\tilde{f}$.

Denote $n=\#\left[\tilde{f}^{-1}\left(\frac{1}{2}\right)\right]$ and suppose that $n>1$. Our object is to reduce $n$. Let $F$ be a gradient-like Morse-Smale vector field of $X$ for the function $\left.\widetilde{f}\right|_{X}$. It follows from Morse theory that the union of $\left.\widetilde{f}\right|_{X}$-positive boundary components $\partial_{+} X$ with the set of trajectories that start at saddle critical points of $\left.\widetilde{f}\right|_{X}$ and finish at $\partial_{+} X$ is a strong deformation retract of $X$. Since $X$ is connected, we see that there exists a saddle critical point $z$ of $\left.\widetilde{f}\right|_{X}$ such that the trajectories starting from $z$ finish at different components of $\partial_{+} X$. We denote these trajectories by $\omega_{1}$ and $\omega_{2}$.

Then (Milnor [MJ1], Theorem 4.1) there exists a $\Sigma$-homotopy $\widetilde{f}_{t}$ of $\widetilde{f}_{0}=\left.\widetilde{f}\right|_{X}$ that changes $\left.\widetilde{f}\right|_{X}$ only in an arbitrary small neighborhood of $\left(\omega_{1} \cup \omega_{2}\right) \cap \widetilde{f}^{-1}\left(\frac{1}{4}, \frac{1}{2}\right]$ such that $\frac{1}{2}<\widetilde{f}_{1}(z)<1$, but $\widetilde{f}_{1}\left(z^{\prime}\right)<\frac{1}{2}$ for all other critical point $z^{\prime}$ of $\tilde{f}_{1}$. It follows that $\frac{1}{2}$ is a regular value for $\widetilde{f}_{1}$ and the level-set $\tilde{f}_{1}^{-1}\left(\frac{1}{2}\right)$ has precisely $n-1$ connected components. Now (3) follows by induction on $n$.

Lemma 3.2. Every Morse mapping $f: M \rightarrow S^{1}$ is $\Sigma$-homotopic to a Morse mapping $g$ such that for some regular value $c$ of $g$ we have:

(A) if $f$ is null-homotopic, then $g^{-1}(c)=\varnothing$;

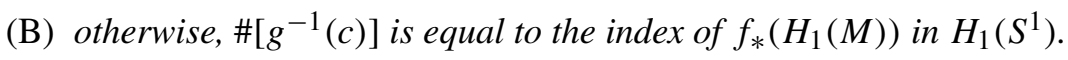

Proof. Let $c$ be a regular value of $f$ such that $f^{-1}(c) \cap \partial M=\varnothing$ and let $n=$ $\#\left[f^{-1}(c)\right]$. We cut $M$ and obtain the surface $\widetilde{M}=\widetilde{M}(f, c)$ and the function $\tilde{f}: \widetilde{M} \rightarrow$ $[0,1]$ as above.

By Lemma 3.1, if $Q_{0} \cup Q^{1} \neq \varnothing$ or if for some connected component $X$ of $Q_{0}^{1}$ the intersection $X \cap B_{0}$ has more than one component, then there exists a $\Sigma$-homotopy $\tilde{f}_{t}$ of $\tilde{f}$ with respect to some neighborhood of $B$ such that $\#\left[\tilde{f}_{1}^{-1}\left(\frac{1}{2}\right)\right]<n$. As noted above, this $\Sigma$-homotopy yields a $\Sigma$-homotopy $f_{t}$ of $f=f_{0}$ to a Morse mapping $f_{1}$ with respect to some neighborhood of $f^{-1}(c)$ such that $\#\left[f_{1}^{-1}\left(c_{1}\right)\right]<n$, where $c_{1}=q\left(\frac{1}{2}\right)$ is a regular value of $f_{1}$.

Repeating these arguments for $f_{1}$ and $c_{1}$, and using induction on $n$ we will obtain a Morse mapping $f_{k}$ and its regular value $c_{k}$ such that either (i) $f_{k}^{-1}\left(c_{k}\right)=\varnothing$ or (ii) $Q_{0}\left(f_{k}, c_{k}\right)=Q^{1}\left(f_{k}, c_{k}\right)=\varnothing$ and for every connected component $X$ of $Q_{0}^{1}=\widetilde{M}\left(f_{k}, c_{k}\right)$ the intersection $X \cap B_{i}\left(f_{k}, c_{k}\right)$ is non-empty and connected, whence it is an SCC.

Suppose that $f_{k}$ is null-homotopic. Then $f_{k}$ lifts to a Morse function $\widetilde{f}_{k}: M \rightarrow$ $\mathbb{R}^{1}$ which must have a global minimum and maximum. Therefore, if $f_{k}^{-1}\left(c_{k}\right) \neq$ $\varnothing$ (case (ii)), then $Q_{0}\left(f_{k}, c_{k}\right) \cup Q^{1}\left(f_{k}, c_{k}\right) \neq \varnothing$, which contradicts (ii). Hence, $f_{k}^{-1}\left(c_{k}\right)=\varnothing$. This proves (A). 
Suppose $f_{k}$ is not null-homotopic. For convenience we denote $f_{k}$ by $f$ and $c_{k}$ by $c$. We will now lift $f$ onto the covering of $S^{1}$ corresponding to the subgroup $f\left(H_{1}(M)\right)$ of $H_{1}\left(S^{1}\right)$. Let $m=\#[\widetilde{M}]$ and let $p_{m}: S^{1} \rightarrow S^{1}$ be the $m$-sheet-covering of $S^{1}$ defined by the formula $p_{m}\left(e^{2 \pi i t}\right)=e^{m 2 \pi i t}, t \in[0,1]$.

First notice, that the set of connected components of $\widetilde{M}$ admits a natural cyclic ordering. Indeed, let $X_{0}$ be any component of $\tilde{M}$. If $X_{k},(k \geq 0)$ is defined, then there exists a unique connected component $X_{k+1}$ of $\widetilde{M}$ such that $p\left(X_{k+1} \cap B_{0}\right)=$ $p\left(X_{k} \cap B_{1}\right)$. Since $M$ is connected, it follows that every connected component of $\tilde{M}$ is numbered in this way.

Then the following formula defines a lifting $\bar{f}: M \rightarrow S^{1}$ of $f$ onto the $m$-sheet covering of $S^{1}$ :

$$
\bar{f}(x)=\exp \frac{2 \pi i}{m}\left(\tilde{f}\left(p^{-1}(x)\right)+k\right), \quad x \in p\left(X_{k}\right), k=0, \ldots, m-1,
$$

i.e. $p_{m} \circ \bar{f}=f$.

Finally, let us prove that the homomorphism $\bar{f}_{*}: H_{1}(M) \rightarrow H_{1}\left(S^{1}\right)$ is onto. This will imply that the index of $f\left(H_{1}(M)\right)$ in $H_{1}\left(S^{1}\right)$ is $m$. For every $k=0, \ldots, m-1$ let $\omega_{k}:[0,1] \rightarrow X_{k}$ be a simple arc which is transversal to level-sets of $\widetilde{f}$ and such that $\tilde{f}\left(\omega_{k}(t)\right)=t, p\left(\omega_{k}(1)\right)=p\left(\omega_{k+1}(0)\right)$ and $p\left(\omega_{m-1}(1)\right)=p\left(\omega_{0}(0)\right)$. Evidently, these arcs constitute an SCC $\omega$ on $M$ such that the restriction $\left.\bar{f}\right|_{\omega}$ is a homeomorphism, whence $\bar{f}_{*}$ is onto. Thus (B) is proved.

3.1. Orientation of level-sets of $\boldsymbol{f}$. Suppose that $M$ is orientable. Let $c \in S^{1}$ be a regular value of a Morse mapping $f: M \rightarrow S^{1}, L=f^{-1}(c)$ the corresponding level-set of $f$, and $F$ a gradient vector field for $f$ taken in some Riemannian metric on $M$. Then the orientation of $M$ together with $F$ yields an orientation of $L$ so that the homology class of an oriented cycle $\left[f^{-1}(c)\right] \in H_{1}(M, \partial M)$ does not depend on a particular choice of a regular value $c$ and even on the homotopy class of $f$. For every $x \in L$ let $v_{x}$ be a tangent vector to $L$ at $x$ such that the pair $\left(v_{x}, \operatorname{grad} f(x)\right)$ gives a positive orientation of $M$. Then the orientation of $L$ defined by $v_{x}$ satisfies the conditions of the previous sentence.

Let $\xi \in H^{1}\left(S^{1}\right)$ be a generator that defines the positive orientation of $S^{1}$ and let $\omega$ be an intersection form on $H_{1}(M, \partial M)$. Then for every oriented SCC $\gamma: S^{1} \rightarrow M$, regarded as an element of $H_{1}(M)$, we have

$$
f(\xi)(\gamma)=\langle L, \gamma\rangle=\operatorname{deg}\left(\left.f\right|_{\gamma}\right) .
$$

Since $f$ is constant on boundary components of $M$ and is not null-homotopic it follows that $f(\xi) \neq 0$ in $H^{1}(M, \partial M)$. The intersection form $\omega$ on $M$ yields an isomorphism $\phi: H^{1}(M, \partial M) \rightarrow H_{1}(M, \partial M)$ which by Eq. (3.4) maps $f(\xi)$ to the homology class $[L]$. 
In particular, if $h: M \rightarrow M$ is a diffeomorphism such that $f \circ h$ and $f$ are homotopic, then it follows that $h^{*}(f(\xi))=f(\xi)$ in $H^{1}(M, \partial M)$ and $h_{*}([L])=[L]$ in $H_{1}(M, \partial M)$.

\section{Kronrod-Reeb graph of a Morse mapping}

Let $f: M \rightarrow P$ be a Morse mapping, $c \in P$, and $\gamma$ a connected component of $f^{-1}(c)$. We call $\gamma$ regular if it contains no critical points of $f$; otherwise $\gamma$ is critical.

Consider the partition of $M$ by the connected components of level-sets of $f$. The factor-space $\Gamma_{f}$ of $M$ by this partition has the structure of a one-dimensional CWcomplex and is called the Kronrod-Reeb graph or KR-graph of $f$ (see e.g. [KA], [KE], [SV2]). There is a unique decomposition

$$
f: M \stackrel{f^{*}}{\longrightarrow} \Gamma_{f} \stackrel{f_{\Gamma}}{\longrightarrow} P,
$$

where $f^{*}$ is a factor map and for every open edge $e$ of $\Gamma_{f}$ the restriction $\left.f_{\Gamma}\right|_{e}$ is a local homeomorphism. Notice that the orientation of $P$ yields a unique orientation of $e$ preserved by $f_{\Gamma}$. The mapping $f_{\Gamma}$ will be called KR-map associated with $f$.

The vertices of $\Gamma_{f}$ correspond to the critical components of level-sets of $f$ and to the boundary circles of $M$. The last type of vertices will be denoted on the KR-graph by circles $\circ$ (see e.g. Figure 4.1). Notice that for non-orientable surfaces, KR-graphs can possess vertices of degree 2 (e.g. [KE]). We will denote these vertices by stars $*$.

Let $f, g: M \rightarrow P$ be Morse mappings. By an isomorphism between their KRgraphs we will mean a homeomorphism $\Gamma_{g} \rightarrow \Gamma_{f}$ preserving orientations of edges and the sets of $\circ$ - and $*$-vertices.

We will say that their KR-maps $f_{\Gamma}$ and $g_{\Gamma}$ are equivalent provided there exist a preserving orientation diffeomorphism $\phi$ of $P$ and an isomorphism $\alpha: \Gamma_{g} \rightarrow \Gamma_{f}$ such that in the following diagram the right square is commutative:

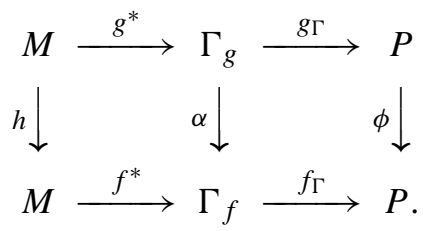

The mappings $f$ and $g$ are said to be equivalent provided there exists a diffeomorphism $h$ of $M$ such that $f \circ h=\phi \circ g$. In this case there is a unique equivalence $\alpha$ between KR-maps of $f$ and $g$ such that the whole diagram (4.1) is commutative.

A Morse mapping $f$ is called generic if every level-set of $f$ contains at most one critical point. Let $f$ be a generic Morse mapping. If $M$ is orientable, then the degree 
of each vertex of $\Gamma_{f}$ is either 1 or 3 . If $M$ is non-orientable, then $\Gamma_{f}$ may possess vertices of degree 2 .

The following lemma is well-known. Its different variants can be found in $[\mathrm{BF}]$, [KE], [K], [SV2].

Lemma 4.1. Two generic Morse mappings $f$ and $g$ having equivalent KR-maps are equivalent.

We say that a Morse mapping $f$ is canonical if its KR-map is equivalent to that drawn in Figures 4.1 or 4.2 .

First consider the case $P=\mathbb{R}$, see Figure 4.1. The part of KR-graph under the rectangle corresponds to the following cases of $M$ :

a) $M$ is orientable.

b) $M$ is non-orientable of odd genus $g$.

c) $M$ is non-orientable of even genus $g$.

d) $M$ is non-orientable, $g \geq 3$ and is odd. In this case we will use two types of canonical Morse functions shown in Figure 4.1. They are related by a $\Sigma$-homotopy, see [KE]. For the case $P=S^{1}$ a canonical Morse mapping $f: M \rightarrow S^{1}$ can be

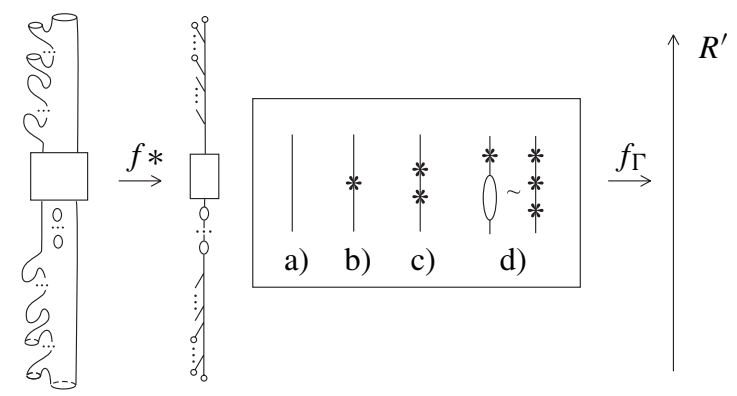

Figure 4.1. KR-graphs and KR-maps of a canonical Morse function $M \rightarrow \mathbb{R}$.

described as follows: there is a regular value $c$ of $f$ such that $\gamma=f^{-1}(c)$ is an SCC. Moreover, if we cut $M$ along $\gamma$, then the restriction of $f: M \backslash \gamma \rightarrow S^{1} \backslash c$ is a canonical Morse function. Its KR-graph is hidden behind the rectangle, see Figure 4.2.

Notice also that a canonical Morse mapping is generic and the homomorphism $f_{*}: H_{1}(M) \rightarrow H_{1}\left(S^{1}\right)$ is onto.

Lemma 4.2. Let $f, g: M \rightarrow P$ be two canonical Morse mappings of the same critical type $K(f)=K(g)$. Then they are equivalent.

Moreover, there is a $\Sigma$-homotopy of $g$ to a canonical Morse mapping $g_{1}$ such that $g_{1}=f \circ h$, where $h$ is a diffeomorphism of $M$. 


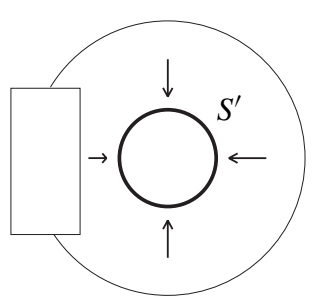

Figure 4.2. KR-graphs and KR-maps of a canonical Morse mapping $M \rightarrow S^{1}$.

Proof. Evidently, KR-graph and KR-map of a canonical Morse mapping is determined by the numbers $c_{0}, c_{2}, b_{+}, b_{-}$and the (orientable or non-orientable) genus $g$ of $M$. Notice that $c_{1}$ is related to these numbers via Euler characteristic.

Hence the condition $K(f)=K(g)$ implies that KR-maps of $f$ and $g$ are equivalent. Then by Lemma 4.1, $f$ and $g$ are equivalent, i.e. $p \circ g=f \circ h$, where $p$ is a preserving orientation diffeomorphism of $P$ and $h$ is a diffeomorphism of $M$. It follows that $p$ is isotopic to $\operatorname{id}_{M}$. Let $p_{t}$ be an isotopy of $p=p_{1}$ to $\mathrm{id}_{M}=p_{0}$. Then $g_{t}=p_{t} \circ g$ is a $\Sigma$-homotopy of $g=g_{0}$ to $g_{1}=p_{1} \circ g=p \circ g=f \circ h$.

\section{Reduction of the problem}

Let $f, g: M \rightarrow P$ be two Morse mappings such that $K(f)=K(g)$. We have to prove that $f \stackrel{\Sigma}{\sim} g$.

In this section we reduce the proof of the Main Theorem to the case when $f$ and $g$ are canonical, and $g=f \circ h$, where $h$ is a diffeomorphism of $M$. This was done in $[\mathrm{KE}]$ for the case $P=\mathbb{R}$. Let $P=S^{1}$.

5.1. Step 1. It may be assumed that the homomorphism $f_{*}=g_{*}: H_{1}(M) \rightarrow$ $H_{1}\left(S^{1}\right)$ is surjective. In particular, $f$ and $g$ are not null-homotopic. This also implies that $M$ is neither a sphere nor a projective plane (with holes if $\partial M \neq \varnothing$ ).

Indeed, suppose that the homomorphism $f_{*}=g_{*}$ is not onto. Let $p: \widetilde{S} \rightarrow S^{1}$ be the covering of $S^{1}$ corresponding to the subgroup $f_{*}\left(H_{1}(M)\right) \subset H_{1}\left(S^{1}\right)=\pi_{1}\left(S^{1}\right)$ and let $\widetilde{f}, \widetilde{g}: M \rightarrow \widetilde{S}$ be some liftings of $f$ and $g$ respectively which are evidently Morse.

Lemma 5.1. $f \stackrel{\Sigma}{\sim} g$ if and only if $\tilde{f} \stackrel{\Sigma}{\sim}$.

The proof is easy and is left to the reader. It can be found in $[\mathrm{M}]$. 
5.2. Step 2. We may assume that $f$ and $g$ are canonical due to the following statement:

Proposition 5.2 ([KE]). Every Morse mapping $f: M \rightarrow P$ such that the homomorphism $f_{*}\left(H_{1}(M)\right) \subset H_{1}\left(S^{1}\right)=\pi_{1}\left(S^{1}\right)$ is onto is $\Sigma$-homotopic to a canonical one.

It follows from this proposition that $f \stackrel{\Sigma}{\sim} f_{1}$ and $g \stackrel{\Sigma}{\sim} g_{1}$, where $f_{1}$ and $g_{1}$ are canonical Morse mappings of the same critical type $K(f)=K(g)$. Then by Lemma $4.2 g_{1}=f_{1} \circ h$, where $h$ is a diffeomorphism of $M$.

Proof. As noted above, this statement is proved in [KE] (Lemma 10) for closed surfaces and $P=\mathbb{R}$. The proof easily extends to surfaces with boundary. Suppose that $P=S^{1}$. Since $f_{*}$ is onto, it follows from Lemma 3.2, that $f$ is $\Sigma$-homotopic to a Morse mapping $f_{1}$ such that $\alpha=f_{1}^{-1}(c)$ is an SCC, where $c$ is a regular value of $f_{1}$. Cutting $M$ along $\alpha$ as in Section 3 we obtain a surface $\widetilde{M}$ and a function $\tilde{f}: \widetilde{M} \rightarrow[0,1]$. Then by the $\mathbb{R}$-case of this proposition $\tilde{f}$ is $\Sigma$-homotopic with respect to a neighborhood of $B$ to a canonical Morse function. This $\Sigma$-homotopy yields a $\Sigma$-homotopy of $f$ to a canonical Morse mapping.

\section{Admissible diffeomorphisms and curves}

Definition 6.1. Let $f: M \rightarrow P$ be a Morse mapping. A diffeomorphism $h: M \rightarrow M$ will be called $f$-admissible provided $f \circ h$ is $\Sigma$-homotopic to $f$. Notice that $f$ admissibility implies that $h$ preserves the sets of $f$-positive and $f$-negative components of $\partial M$ and that $f$ and $f \circ h$ are homotopic.

Let $\mathcal{A}(f) \subset \mathscr{D} M$ be the set of all $f$-admissible diffeomorphisms, let $\mathcal{D}_{\text {id }} M$ be the identity component of $\mathscr{D} M$, and let $C(f)$ be the path-component of $f$ in $\mathcal{F}(M, P)$.

Lemma 6.2. $\mathcal{A}(f)$ is a group consisting of full isotopy classes, i.e. $\mathcal{D}_{\mathrm{id}} M \subset \mathcal{A}(f)$. Moreover, if $g \in C(f)$, then $\mathcal{A}(g)=\mathcal{A}(f)$.

Proof. Suppose that $p, q \in \mathcal{A}(f)$ and let $f \stackrel{\Phi_{t}}{\sim} f \circ p$ and $f \stackrel{\Psi_{t}}{\sim} f \circ q$ be $\Sigma$ homotopies. Then $p \circ q$ and $p^{-1} \in \mathcal{A}(f)$. Indeed,

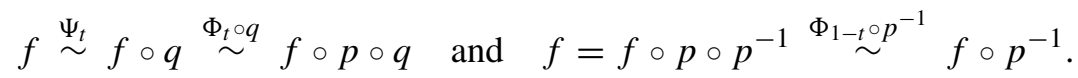

Thus $\mathcal{A}(f)$ is a group.

If $p \stackrel{H_{t}}{\sim} p_{1}$ is an isotopy, then the homotopy $f \stackrel{\Phi_{t} \circ H_{t}}{\sim} f \circ p_{1}$ is a $\Sigma$-homotopy. Thus $\mathcal{A}(f)$ consists of full isotopy classes. 
Finally, if $f \stackrel{\Psi_{t}}{\sim} g$ is a $\Sigma$-homotopy, then $g \stackrel{\Psi_{t}}{\sim} f \stackrel{\Phi_{t}}{\sim} f \circ p \stackrel{\Psi_{1-t} \circ p}{\sim} g \circ p$. Hence $p \in \mathcal{A}(g)$, i.e. $\mathcal{A}(f) \subset \mathcal{A}(g)$. Similarly $\mathcal{A}(g) \subset \mathcal{A}(f)$.

We will now consider three types of "elementary diffeomorphisms" and show that they preserve certain simple Morse functions.

6.1. Dehn twists. Let $\gamma$ be a two-sided oriented SCC in $M$. For the definition of a Dehn twist along $\gamma$ see e.g. [D], [L1]. This diffeomorphism is supported in some neighborhood of $\gamma$ and its effect on such a neighborhood is shown in Figure 6.1a).

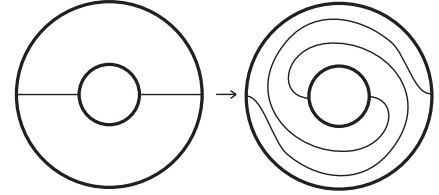

a)

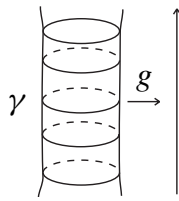

b)

Figure 6.1. Dehn twist.

Definition 6.3. Let $\gamma$ be a two-sided SCC in $M \backslash \partial M$. We say that $\gamma$ is $f$-admissible if $f$ is $\Sigma$-homotopic to a Morse mapping $g$ such that $\gamma$ is a connected component of a regular level-set of $g$.

Lemma 6.4. Let $\gamma \subset \operatorname{Int} M$ be an $f$-admissible oriented SCC in $M$. Then a Dehn twist $t_{\gamma}$ along $\gamma$ is $f$-admissible.

Proof. Let $f \stackrel{F}{\sim} g$ be a $\Sigma$-homotopy such that $\gamma$ is a connected component of a regular level-set of $g$. We will construct a Dehn twist $t_{\gamma}$ along $\gamma$ such that $g=g \circ t_{\gamma}$. Then $t_{\gamma}$ is $g$-admissible, whence by (1) of Lemma $6.2 t_{\gamma}$ is also $f$-admissible.

Since $\gamma$ is a regular component of a level set of $g$, there is a regular neighborhood of $\gamma$ which is diffeomorphic to $S^{1} \times I$ and such that the function $g$ is the projection to $I$, see Figure 6.1b). Then there is a Dehn twist $t_{\gamma}$ along $\gamma$ that preserves the sets of the form $S^{1} \times\{t\}$. They are level-sets of $g$, whence $t_{\gamma}$ preserves $g$.

6.2. Boundary slides. Let $A$ be an annulus and let $C_{0}, C_{1}$ be the connected components of $\partial A$. Divide $C_{1}$ into four arcs of equal length $l_{1}, \ldots, l_{4}$ so that $l_{1}$ is opposite to $l_{3}$ and $l_{2}$ to $l_{4}$. Let us identify the opposite points of $l_{1}$ and $l_{3}$. Then the quotient is a Möbius strip $B$ with the hole $C_{1}^{\prime}=l_{2} \cup l_{4}$.

Let $\tau: A \rightarrow A$ be a half-Dehn twist along $C_{1}$, which exchanges $l_{1}$ with $l_{3}$ and $l_{2}$ with $l_{4}$ and is the identity near $C_{0}$. Then $\tau$ yields a certain diffeomorphism $v$ of $B$ that "rotates $C_{1}^{\prime}$ by $\pi$ and fixes $C_{0}$ ", see Figure 6.2a). 
Suppose that $B$ is embedded into $M$ so that $C_{1}$ is mapped onto a connected component $C$ of $\partial M$. Then $v$ extends by the identity on all of $M$. This diffeomorphism is called a boundary slide of $C$ along $B$.

Notice that our description of boundary slide differs from ones given in [KM], [SB]. The advantage is an evidence of the symmetry of $v$.

Now it is easy to see that there is a Morse function $f: B^{\prime} \rightarrow[0,1]$ having a unique critical point of index 1 and such that $f^{-1}(0)=C_{0}, f^{-1}(1)=C_{1}^{\prime}$. Its critical level sets and the KR-graph are shown in Figure 6.2b).

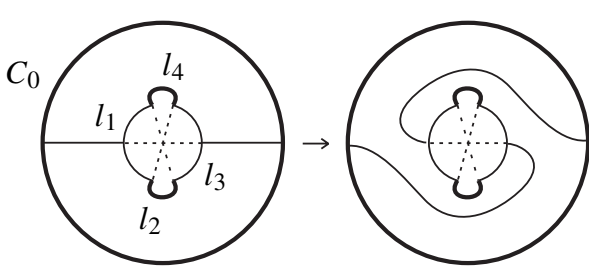

a)

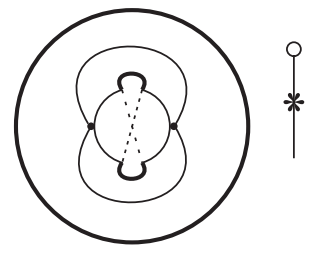

b)

Figure 6.2. Boundary slide.

The following lemma is obvious.

Lemma 6.5. $f: M \rightarrow P$ be a Morse mapping on a non-orientable surface $M$. Suppose that the KR-graph of $f$ has an edge e such that one of its vertices, $v_{1}$, has degree 2 and another one, $v_{2}$, corresponds to the boundary component of $M$, see Figure 6.2b). Let $N$ be a neighborhood of e containing no vertices of $\Gamma_{f}$ but $\partial e$. Then $B=f_{\Gamma}^{-1}(N) \subset M$ is a Möbius band with hole and there exists a boundary slide v: $M \rightarrow M$ of $f_{\Gamma}^{-1}\left(v_{2}\right)$ along $B$ such that $f \circ y=f$.

6.3. Crosscap slides. This type of diffeomorphisms was introduced by W. B. R. Lickorish [L2] and called a $Y$-diffeomorphism. In [KM], [SB] the term crosscap slide is used. We recall the definition of this diffeomorphism (given in $[\mathrm{BC}]$ ) via oriented double coverings.

Let $K$ be a Klein bottle with two holes and let $p: T \rightarrow K$ be its oriented double covering, where $T$ is a torus with 4 holes. We can assume that $T$ is embedded into $\mathbb{R}^{3}$ so that it is symmetrical with respect to the origin 0 . In other words it is invariant under the involution $\xi(x, y, z)=(-x,-y,-z)$ of $\mathbb{R}^{3}$, see Figure 6.3a).

Let $V_{1}, \ldots, V_{4}$ be the connected components of $\partial T$ numbered so that $\xi\left(V_{1}\right)=V_{2}$ and $\xi\left(V_{3}\right)=V_{4}$. Then there is a diffeomorphism $\tilde{y}: T \rightarrow T$ which is fixed near $V_{3} \cup V_{4}$, coincides with $\xi$ near $V_{1} \cup V_{2}$ and such that $\tilde{y} \circ \xi=\xi \circ \tilde{y}$. Thus $y$ can be described as a "rotation" of $T$ with respect to the $z$-axis by $\pi$ with fixed boundary components $V_{3}$ and $V_{4}$. For example, in Figure 6.3a) an arc and its image under $\xi$ are shown. It follows that $\tilde{y}$ induces some diffeomorphism $y$ of $K$ fixed near $\partial K$. 
Suppose that $K \subset M$ is embedded into $M$. Then $y$ extends by the identity to a diffeomorphism of $M$. Such a diffeomorphism of $M$ is called $Y$-diffeomorphism or crosscap slide based in $K$.

Notice that there is a Morse function $\tilde{f}: T \rightarrow \mathbb{R}$ with 4 critical points such that $\tilde{f} \circ \tilde{y}=\tilde{f}$, see Figure 6.3a), where the critical level-sets of $\widetilde{f}$ are shown. Then $\tilde{f}$ yields a unique Morse function $f: K \rightarrow \mathbb{R}$ having 2 critical points and such that $f \circ y=f$. The KR-graphs $\Gamma_{\tilde{f}}$ and $\Gamma_{f}$ of $\tilde{f}$ and $f$ are shown in Figure 6.3b).

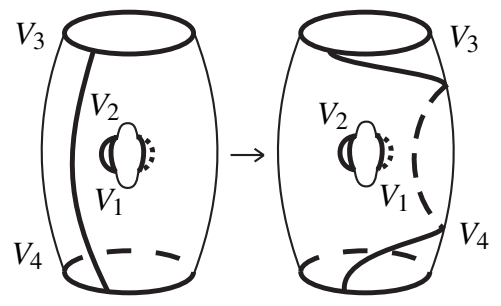

a)

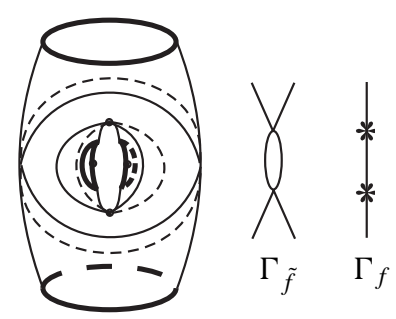

b)

Figure 6.3. Crosscap slide on the orientable covering.

Lemma 6.6. Let $f: M \rightarrow P$ be a Morse mapping on a non-orientable surface $M$. Suppose that the KR-graph of $f$ has an edge e with vertices of degree 2. Let $N$ be a neighborhood of e containing no vertices of $\Gamma_{f}$ but $\partial e$. Then $K=f_{\Gamma}^{-1}(N) \subset M$ is a Klein bottle with two holes and there exists a Y-diffeomorphism y: $M \rightarrow M$ based in $K$ such that $f \circ y=f$.

\section{Mapping class group of a surface with boundary}

Let $\widehat{M}$ be a closed connected surface and let $X=\left\{x_{1}, \ldots, x_{n}\right\}$ be a set of mutually distinct points of $\widehat{M}$. The extended mapping class group $\mathcal{M}_{n}(M)$ of $M$ is defined to be the group of isotopy classes of diffeomorphisms of $\widehat{M}$ which take $X$ to itself. The pure extended mapping class group $\mathcal{P \mathcal { M }}_{n}(M)$ of $M$ is the group of isotopy classes of diffeomorphisms of $\widehat{M}$ which take $X$ point-wise. The groups $\mathcal{M}_{0}(\widehat{M})$ and $\mathcal{P} \mathcal{M}_{0}(\widehat{M})$ will be denoted by $\mathcal{M}(\widehat{M})$ and $\mathcal{P} \mathcal{M}(\widehat{M})$ respectively.

Let $M$ be a connected surface with boundary $\partial M$ consisting of $n$ connected components $V_{1}, \ldots, V_{n}$. Regarding these components as punctures, we can identify the groups $\mathcal{M}(M)$ and $\mathcal{P} \mathcal{M}(M)$ with $\mathcal{M}_{n}(\widehat{M})$ and $\mathcal{P} \mathcal{M}_{n}(\widehat{M})$.

We recall the sets of generators of $\mathcal{M}(M)$ and $\mathcal{P} \mathcal{M}(M)$ given in [B2], [G] for orientable surfaces and in $[\mathrm{KM}]$ for nonorientable ones. 
7.1. Orientable case. Suppose that $M$ is orientable. Consider the following 3 types of diffeomorphisms of $M$ :

(1) Let $O$ be a reversing orientation diffeomorphism of $M$.

(2) Let $\alpha_{i}, \beta_{i}, \gamma_{i}, \delta_{i}, \epsilon_{i}$ be the SCC shown in Figures 7.1a), where the bold points denote connected components of $\partial M$ divided into two parts (positive and negative components). We will refer to them as SCCs of configuration $\mathcal{C}$. Denote by $t_{\alpha_{i}}, t_{\beta_{i}}$, $t_{\gamma_{i}}, t_{\delta_{j}}, t_{\epsilon_{i}}$ the corresponding Dehn twists.

(3) For every pair $i<j=1, \ldots, n$ let $\sigma_{i j}$ be an SCC that separates $M$ into two connected components so that one of them is a sphere $S$ with 3 holes whose boundary components are $\sigma_{i j}$ and the connected components $V_{i}$ and $V_{j}$ of $\partial M$, see Figure 7.1b). Let $b_{i j}$ be a diffeomorphism of $M$ with support in $S$ which permutes boundary components $V_{i}$ and $V_{j}$ and preserves all others. Evidently, $b_{i j}^{2}$ is a Dehn twist $t_{\sigma_{i j}}$ along $\sigma_{i j}$.

Theorem 7.1 ([B2], [G]). The group $\mathcal{M}(M)$ is generated by

(i) $\left\{O, b_{i j}: i, j=1, \ldots, n\right\}$ if $g=0$;

(ii) $\left\{t_{l}, O, b_{i j}: l \in \mathcal{C}, i, j=1, \ldots, n\right\}$ if $g \geq 1$.

The group $\mathcal{P} \mathcal{M}(M)$ is generated by

(i) $\left\{O, b_{i j}^{2}=t_{\sigma_{i j}}: i, j=1, \ldots, n\right\}$ if $g=0$;

(ii) $\left\{t_{l}, O: l \in \mathcal{C}, i, j=1, \ldots, n\right\}$ if $g \geq 1$.

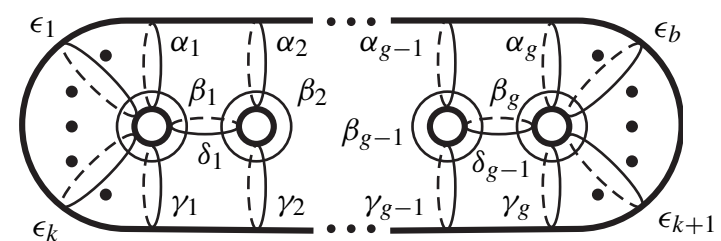

a)

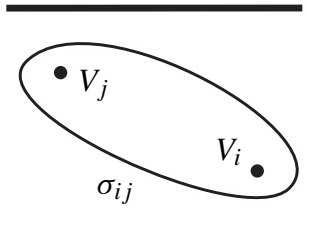

b)

Figure 7.1. The configuration $\mathcal{C}$. Orientable case.

7.2. Generators for $\mathcal{M}(\boldsymbol{M})$. Non-orientable case. Suppose that $M$ is non-orientable of genus $g$, see Figure 7.2, where the interiors of the shaded disks are removed and then the antipodal points on each boundary component are to be identified.

Consider the following 4 types of diffeomorphisms of $M$ :

(1) Let $y$ be a crosscap slide of $M$. If $g \geq 3$, then we additionally assume that $y^{2}$ is a Dehn twist along a two-sided separating SCC both components of whose complement are non-orientable. 
(2) and (3) Similarly to the oriented case we define the configuration $\mathcal{C}$ of SCCs $\alpha_{i}, \beta_{i}, \gamma_{i}, \delta_{i}, \epsilon_{i}$ shown in Figure 7.2, SCCs $\sigma_{i j}$, the corresponding Dehn twists and diffeomorphisms $b_{i j}$.

(4) Let $v_{i}$ denote the boundary slide obtained by sliding the boundary component $V_{i}$ along the loop $\mu$ if $g$ is odd and along $\mu_{1}$ if $g$ is even, see Figure 7.3. Also if $g$ is even, denote by $\omega_{i}$ the boundary slide obtained by sliding $V_{i}$ once along the loop $\mu_{2}$.

Theorem $7.2([\mathrm{KM}])$. The group $\mathcal{M}(M)$ is generated by

(i) $\left\{v_{k}, b_{i j}: i, j, k=1, \ldots, n, i<j\right\}$ if $g=1$;

(ii) $\left\{t_{\beta_{0}}, y, v_{k}, b_{i j}: i, j, k=1, \ldots, n, i<j\right\}$ if $g=2$;

(iii) $\left\{t_{l}, y, v_{k}, b_{i j}: l \in \mathcal{C}, i, j, k=1, \ldots, n, i<j\right\}$ if $g \geq 3$ is odd;

(iv) $\left\{t_{l}, y, v_{k}, \omega_{k}, b_{i j}: l \in \mathcal{C}, i, j, k=1, \ldots, n, i<j\right\}$ if $g \geq 4$ is even.

Replacing every $b_{i j}$ by $b_{i j}^{2}=t_{\sigma_{i j}}$ we obtain generators for $\mathcal{P} \mathcal{M}(M)$.
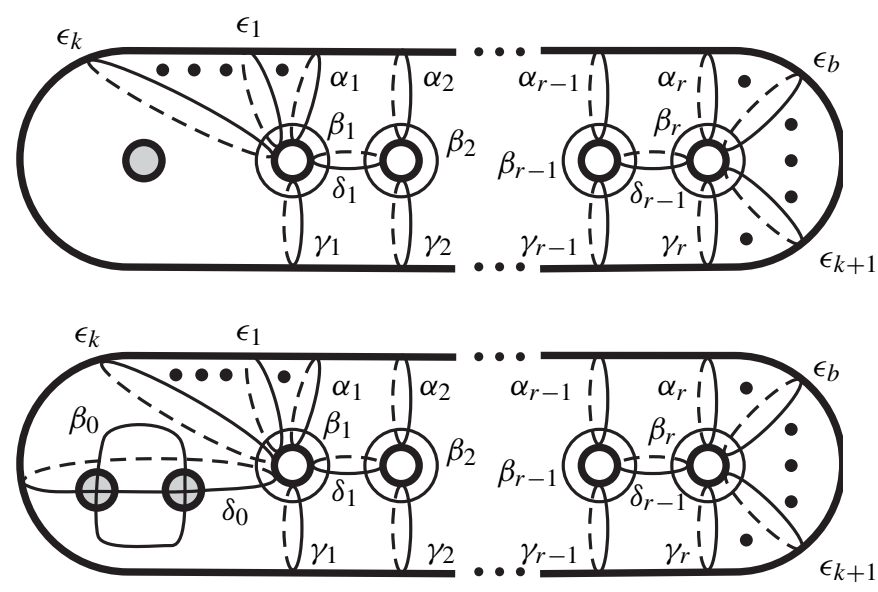

Figure 7.2. The configuration $\mathcal{C}$ for $g=2 r+1$ and $g=2 r+2$. Non-orientable case.
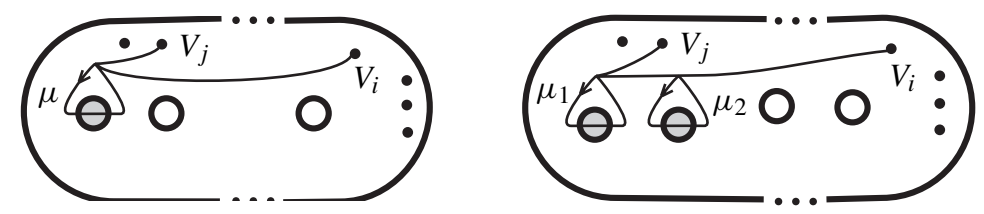

Figure 7.3. Boundary slides for $g=2 r+1$ and $g=2 r+2$. 
7.3. Generators of $\mathcal{M}(M)$ for canonical Morse mapping. Given a Morse mapping $f$, denote by $\mathcal{M}_{f}(M)$ the subgroup of $\mathcal{M}(M)$ consisting of diffeomorphisms that preserve the sets of $f$-positive and $f$-negative components of $\partial M$. Evidently, $\mathcal{A}(f) \subset \mathcal{M}_{f}(M)$.

Lemma 7.3. Let $f: M \rightarrow P$ be a canonical Morse mapping. In the case $P=S^{1}$ assume that $M$ is orientable. Then there is a "canonical" set of generators for $\mathcal{M}_{f}(M)$ such that

(i) for the case $P=\mathbb{R}^{1}$ all of them are $f$-admissible, i.e. $\mathcal{A}(f)=\mathcal{M}_{f}(M)$, whence the Main Theorem holds for this case;

(ii) for $P=S^{1}$ (and orientable $M$ ) all but one of them are also $f$-admissible.

Remark 7.4. Recall that we do not give the proof of the Main Theorem (by the new method) for the case that $M$ is non-orientable and $P=S^{1}$. Therefore we also do not consider this case in Lemma 7.3 since it is more complicated and due to the length of the paper, see also the last paragraph of this section.

Proof. Let $f$ be a canonical Morse mapping. We will construct a set of generators for $\mathcal{M}(M)$ described in Theorems 7.1 and 7.2 such that their $f$-admissibility is rather evident.

First suppose $M$ that is orientable and embedded into $\mathbb{R}^{3}$ as it is shown in Figure 4.1. Then the canonical Morse mapping $f$ is just the projection onto the vertical line.

(1) Let $O$ be a diffeomorphism of $M$ that is a symmetry with respect to the plane of this sheet. Then $O$ reverses orientation of $M$ and preserves $f$, i.e. $f=f \circ O$. Thus $O$ is $f$-admissible.

(2) Comparing Figures 4.1 and 7.1 we see that $\alpha_{i}$ and $\gamma_{i}$ are regular components of regular level-sets of $f$, whence the Dehn twists $t_{\alpha_{i}}$ and $t_{\gamma_{i}}$ are admissible. In Figure 7.4 an $f$-admissibility of twists $t_{\beta_{i}}, t_{\delta_{i}}$ and $t_{\epsilon_{i}}$ is shown.
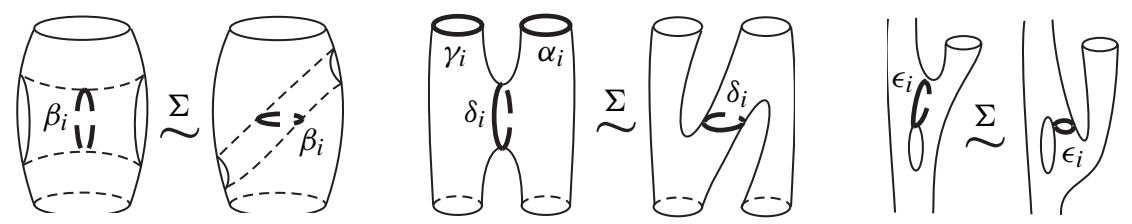

Figure 7.4. $f$-admissibility of configuration $\mathcal{C}$.

(3) Let $V_{i}$ and $V_{j}$ be two $f$-positive components of $\partial M$. Then $f$ is $\Sigma$-homotopic to a Morse mapping $f_{1}$ such that the KR-graph $\Gamma_{f_{1}}$ of $f_{1}$ includes a subgraph $\Gamma_{1}$ shown in Figure 7.5a). Let $\sigma_{i j}$ be an SCC corresponding to a point $s \in \Gamma_{1}$. Then 
there exists a diffeomorphism $b_{i j}$ of $M_{1}$ that exchanges $V_{i}$ and $V_{j}$, preserves $f_{1}$ and $b_{i j}^{2}$ is a Dehn twist along $\sigma_{i j}$. Then $b_{i j}$ and $\sigma_{i j}$ are $f$-admissible.

Now let $V_{i}$ be $f$-positive and $V_{j}$ be $f$-negative. In this case a diffeomorphism $b_{i j}$ permuting $V_{i}$ and $V_{j}$ is not $f$-admissible, since it does not preserve the sets of $f$-positive and $f$-negative boundary components. Nevertheless we will now show that its square $b_{i j}^{2}=t_{\sigma_{i j}}$ is $f$-admissible. Consider two cases.

(a) Suppose that $f$ has at least one critical point of index either 0 or 2 or a boundary component different from $V_{i}$ and $V_{j}$. Then $f$ is $\Sigma$-homotopic to a Morse mapping $f_{1}$ whose KR-graph $\Gamma_{f_{2}}$ includes a subgraph $\Gamma_{2}$ shown in Figure 7.5b). Then we define $\sigma_{i j}$ to be an SCC corresponding to a point $s \in \Gamma_{2}$. Hence $\sigma_{i j}$ is $f$-admissible.

(b) Otherwise, $f$ has no local extremes and $\partial M=V_{1} \cup V_{2}$. Let $\sigma_{12}$ be an SCC that intersects every $\gamma_{i}$ non trivially but no other SCCs of configuration $\mathcal{C}$, separates $M$ in two components $M_{1}$ and $M_{2}$ such that $M_{1}$ is disk with two holes $V_{1}$ and $V_{2}$, see Figure 7.5c).

We claim that $\sigma_{12}$ is not $f$-admissible. Otherwise the restriction of $f$ to $M_{2}$ must have extremes, which could be taken only on boundary components different from $V_{1}$ and $V_{2}$ or at critical points of indices 0 and 2. But all of them are absent on $M_{2}$.

Nevertheless, it is well-known that a Dehn twist $t_{\sigma_{12}}$ is a product of Dehn twists along SCCs of configuration $\mathcal{C}$ except for $\gamma_{i}$. Hence a Dehn twist $t_{\sigma_{12}}$ is $f$-admissible.

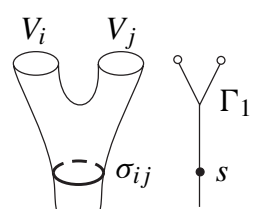

a)

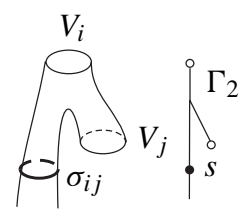

b)

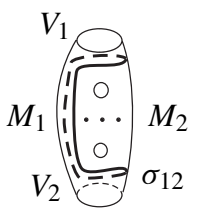

c)

Figure 7.5. $f$-admissibility of $b_{i j}$ and $\sigma_{i j}$.

Suppose that $M$ is non-orientable of genus $g$ (see Figure 7.2) and let $f$ be a canonical Morse mapping as in Figure 4.1. Again we define the generators of $\mathcal{M}(M)$ associated with $f$.

(1) For the case $g \geq 2$ we will now define an $f$-admissible crosscap slide. If $g$ is odd then $\Gamma_{f}$ has an edge $e$ with vertices of degree 2. Otherwise, $f$ is $\Sigma$-homotopic to a Morse function $f_{1}$ whose KR-graph has such an edge, see Figure 4.1d). Then by Lemma 6.6, there exists a crosscap slide $y$ such that $f=f \circ y$ or $f_{1}=f_{1} \circ y$ in the second case. Hence $y$ is $f$-admissible.

Definition and $f$-admissibility of generators of types (2) and (3) are similar to the orientable case. We need to verify the admissibility of $\beta_{0}$ and $\delta_{0}$ for the case $g=2 r \geq 2$.

Let $N$ be a neighborhood of $e$ defined just above containing no vertices of $\Gamma_{f}$ but $\partial e$. Then $K=f_{\Gamma}^{-1}(N) \subset M$ is a Klein bottle with two holes. Let $p: T \rightarrow K$. 
Then $T$ is a torus with four holes. We can assume that the function $\widetilde{f}=f \circ p: T \rightarrow \mathbb{R}$ coincides with the one defined in Section 6.3, see Figure 6.3. Since $\beta_{0}$ and $\delta_{0}$ are two sided, their inverse images $\widetilde{\beta}_{0}=p^{-1}\left(\beta_{0}\right)$ and $\widetilde{\delta}_{0}=p^{-1}\left(\delta_{0}\right)$ in $T$ consist of pair of disjoint SCC. They are shown in Figure 7.6a).

It is shown in Figure 7.6b) that $\widetilde{\beta}_{0}$ is a regular level-set of $\widetilde{f}$. This figure also shows a symmetrical $\Sigma$-homotopy of $\widetilde{f}$ fixed near $\partial T$ which makes $\widetilde{\delta}_{0}$ a regular level-set. Hence $\widetilde{\beta}_{0}$ and $\widetilde{\delta}_{0}$ are $\widetilde{f}$-admissible, whence $\beta_{0}$ and $\delta_{0}$ are $f$-admissible.
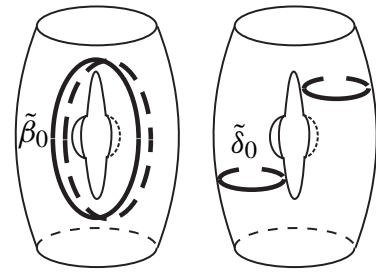

a)

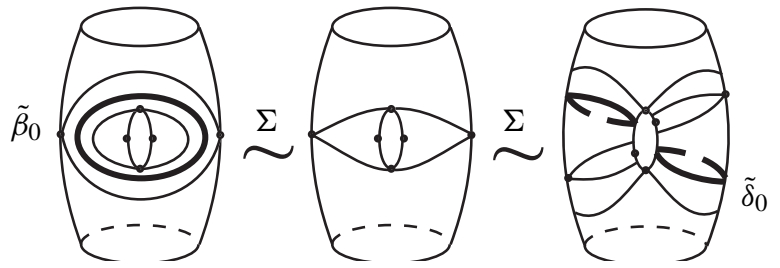

b)

Figure 7.6. $f$-admissibility of $\beta_{0}$ and $\delta_{0}$.

(4) It remains to construct $f$-admissible boundary slides $v_{i}$ and $\omega_{i}$. Let $V_{i}$ be a connected component of $\partial M$ and $z_{i} \in \Gamma_{f}$ be the corresponding o-vertex.

First suppose that $g$ is odd, so $\Gamma_{f}$ has a unique vertex $x$ of degree 2. Then $f$ is $\Sigma$-homotopic to a Morse function $f_{1}$ such that $z_{i}$ and $x$ will be the vertices of the same edge, see Figure 7.7 for the cases when $z_{i}$ is $f$-negative or $f$-positive. Then by Lemma 6.5 , there exists a boundary slide $v_{i}$ of $V_{i}$ preserves $f_{1}$. Whence $v_{i}$ is $f$-admissible.

If $g$ is even, then $\Gamma_{f}$ has two vertices $x_{1}$ and $x_{2}$ of degrees 2 . As in the previous case we define $f$-admissible boundary slices $v_{i}$ for $V_{i}$ and $x_{1}$, and $\omega_{i}$ for $V_{i}$ and $x_{2}$.

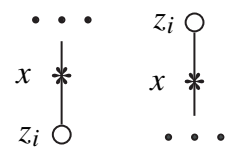

Figure 7.7

Consider now the case $P=S^{1}$. Let $c \in S^{1}$ be a regular value of $f$ and $\alpha_{1}=$ $f^{-1}(c)$ such that the restriction of $f$ to $M \backslash \alpha_{1}$ is a canonical Morse function to $S^{1} \backslash c$.

Suppose that $M$ is orientable. Then the definition of the configuration $\mathcal{C}$ associated with $f$ is shown in Figure 7.8, where $f$ is the "projection" to $\beta_{1}$. Similarly to the previous case we can define a diffeomorphism $O$, Dehn twists along the SCCs of configuration $\mathcal{C}$, and permutations of boundary components $b_{i j}$. The same arguments as in the case $P=\mathbb{R}$ show that all of them are admissible, except for $\beta_{1}$, since $f$ and $f \circ t_{\beta_{1}}$ are not even homotopic. 
If $M$ is non-orientable, then the surface $M \backslash \alpha_{1}$ can be orientable or non-orientable as well. We do not consider this case, see 7.4.

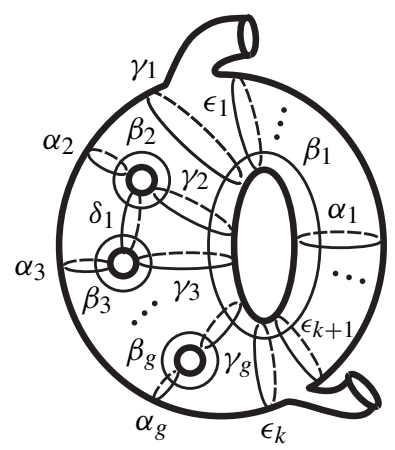

Figure 7.8. Configuration $\mathcal{C}$ if $M$ is orientable and $P=S^{1}$.

\section{Proof of the Main Theorem}

The case $P=\mathbb{R}$ is proved in statement (i) of Lemma 7.3. Before processing with the case $P=S^{1}$ we recall the definition of the Torelli group and its generators.

8.1. Torelli group $\mathcal{T}(\boldsymbol{M})$. Let $M$ be a closed orientable surface. Then the Torelli group of $M$ is a subgroup $\mathcal{T}(M)$ of $\mathcal{P} \mathcal{M}(M)=\mathcal{M}(M)$ consisting of diffeomorphisms of $M$ acting trivially on the homology group $H_{1}(M)$. Evidently, $\mathcal{T}(M)$ is a normal subgroup in $\mathcal{P} \mathcal{M}(M)$.

Suppose now that $\partial M \neq \varnothing$. Let us glue every connected component of $\partial M$ by a 2-disk and denote the obtained closed surface by $\widehat{M}$. Then we obtain an epimorphism $j: \mathcal{P} \mathcal{M}(M) \rightarrow \mathcal{P} \mathcal{M}(\widehat{M})$ induced by the inclusion $M \subset \widehat{M}$, see [B2]. Define the Torelli group $\mathcal{T}(M) \subset \mathcal{P} \mathcal{M}(M)$ of $M$ to be the inverse image $j^{-1}(\mathcal{T}(\widehat{M}))$.

The following theorem describes the generators of ker $j$.

Theorem 8.1 ([B1], [B2]). Let $\alpha_{i}$ and $\beta_{i}$ be the curves of configuration $\mathcal{C}$ on $M$. For every component $V_{j}$ of $\partial M$ let $\alpha_{i k}\left(\beta_{i k}\right)$ be an SCC which together with $\alpha_{i}\left(\beta_{i}\right)$ bounds in $M$ a cylinder with a hole $V_{i}$. Then the kernel of $j$ is generated by the following diffeomorphisms: $s_{i k}=\alpha_{i} \circ \alpha_{i k}^{1}$ and $r_{i k}=\beta_{i} \circ \beta_{i k}^{1}$.

Theorem 8.2 ([B3], [P], [J], [MG]). The Torelli group $\mathcal{T}(M)$ of $M$ is generated by the following types of diffeomorphisms: 
Vol. 80 (2005)

(a) Dehn twists along SCC separating $M$ (if $g=2$ then these diffeomorphisms generate all the group $\mathcal{T}(M),[\mathrm{MG}])$;

(b) products of Dehn twists of the form $t_{\gamma_{1}} \circ t_{\gamma_{2}}^{-1}$, where the SCCs $\gamma_{1}$ and $\gamma_{2}$ are oriented, disjoint, and homologous.

Proof. This theorem was proved for closed surfaces [P] and surfaces with one boundary component $[\mathrm{J}]$. In fact it holds for arbitrary oriented surfaces.

Let $t \in \mathcal{T}(M)$. Since $\widehat{M}$ is closed, we have that $j(t)$ is generated by diffeomorphisms of types (a) and (b). Notice that we can choose the corresponding curves so that they belong to $M$, whence $j(t)$ yields some diffeomorphism $t_{1}$ of surf such that $t_{1}^{-1} \circ t \in \operatorname{ker} j$. By Theorem 8.1, this diffeomorphism is also generated by diffeomorphisms $s_{i k}$ and $r_{i k}$ which evidently are of type (b).

8.2. Proof of the Main Theorem for orientable $M$ and $P=S^{\mathbf{1}}$. It suffices to establish the following statement using the notations of Lemma 7.3.

Proposition 8.3. Let $h \in \mathcal{M}_{f}(M)$ be a diffeomorphism such that the Morse mappings $f$ and $f \circ h: M \rightarrow S^{1}$ are homotopic. Then $h$ is isotopic to a product of diffeomorphisms of the form $p \circ c \circ t$, where

(1) $p$ is generated by $O$ and those $b_{i j}$ that belong $\mathcal{M}_{f}(M)$;

(2) $c$ is generated by Dehn twists along the SCCs of configuration $\mathcal{C}$ but $t_{\beta_{1}}$;

(3) $t \in \mathcal{T}(M)$.

Diffeomorphisms of types (1)-(3) are f-admissible, whence so is $h$.

Proof. Evidently $h$ can be represented as a product $p \circ h_{1}$, where $h_{1} \in \mathcal{P} \mathcal{M}(M)$ and preserves orientation of $M$ and $p$ is of type (1). Then, by Theorem 7.1, $h_{1}$ is generated by the Dehn twists along the curves of configuration $\mathcal{C}$.

Notice that $f$ and $f \circ h_{1}$ are homotopic. This condition will allow us to remove $t_{\beta_{1}}$ from the generators of $h_{1}$ and replace this twist by diffeomorphisms of type (3).

Lemma 8.4. Let $h_{1}$ be a diffeomorphism of $M$ generated by the Dehn twists along the SCCs of configuration $\mathcal{C}$ and such that $f$ and $f \circ h_{1}$ are homotopic. Then there exists an $f$-admissible diffeomorphism c generated by the Dehn twists along the SCCs of configuration $\mathcal{C}$ except for $t_{\beta_{1}}$ such that the diffeomorphism $t=c^{-1} \circ h_{1}$ belongs to $\mathcal{T}(M)$.

Hence it remains to establish that every diffeomorphism $t \in \mathcal{T}(M)$ is $f$-admissible. By Theorem 8.2 it suffices to prove this for diffeomorphisms of type (a) and diffeomorphisms of type (b). 
Theorem 8.5. Let $f: M \rightarrow S^{1}$ be a Morse mapping.

(i) Let $\gamma \subset M$ be an SCC and let $t_{\gamma}$ be a Dehn twist along $\gamma$. Then $t_{\gamma}$ is $f$-admissible if and only if the restriction $\left.f\right|_{\gamma}$ is null-homotopic. If $\gamma$ separates $M$, then $\left.f\right|_{\gamma}$ is null-homotopic, whence every diffeomorphism of type (a) is $f$-admissible.

(ii) Every diffeomorphism of type (b) is $f$-admissible.

Thus in order to complete our proposition, and therefore the Main Theorem, it remains to prove Theorem 8.5 (Sections 12 and 13) and Lemma 8.4 (Section 14).

\section{Symplectic group}

For the proof of Lemma 8.4 we need a description of generators of stabilizers in the symplectic group $\mathrm{Sp}_{2 g}(\mathbb{Z})$. The representation of the group $\mathrm{Sp}_{2 g}(\mathbb{Z})$ is given in [B3]. We will also use the ideas from [OM].

Let $\mathbb{Z}^{2 g}$ be a free $2 g$-module with basis

$$
\alpha_{1}, \ldots, \alpha_{g}, \beta_{1}, \ldots, \beta_{g},
$$

let $I$ be the unity $g \times g$-matrix, and let $e_{i j}$ be a $g \times g$-matrix, whose $(i, j)$-element (the intersection of $i$-th row and $j$-th column) is equal to 1 and all other entries are zeros.

Let also $\omega$ be a skew-symmetric 2 -form whose matrix in the basis (9.1) is the following:

$$
\left(\begin{array}{c|c}
0 & I \\
\hline-I & 0
\end{array}\right)
$$

Thus $\omega\left(\alpha_{i}, \beta_{i}\right)=1$ and $\omega\left(\alpha_{i}, \alpha_{j}\right)=\omega\left(\beta_{i}, \beta_{j}\right)=\omega\left(\alpha_{i}, \beta_{j}\right)=0$ for $i, j=1, \ldots, g$. The group of all linear isomorphisms of $\mathbb{Z}^{2 g}$ preserving $\omega$ is denoted by $\operatorname{Sp}_{2 g}(\mathbb{Z})$ and is called symplectic.

9.1. Transvections. For every $\gamma \in \mathbb{Z}^{2 g}$ the following automorphism $t_{\gamma}$ of $\mathbb{Z}^{2 g}$ defined by the formula

$$
t_{\gamma}(x)=\omega(\gamma, x) \cdot \gamma+x \quad \text { for all } x \in \mathbb{Z}^{2 g}
$$

is called the transvection along $\gamma$. It is easy to see that $t_{\gamma} \in \mathrm{Sp}_{2 g}(\mathbb{Z})$ and

$$
t_{\gamma}^{-1}(x)=-\omega(\gamma, x) \cdot \gamma+x \text { for all } x \in \mathbb{Z}^{2 g} .
$$

Define the following elements of $\mathrm{Sp}_{2 g}(\mathbb{Z})$ :

$$
\begin{gathered}
\mu_{i j}=t_{\alpha_{i}} \circ t_{\alpha_{j}} \circ t_{\alpha_{i}+\alpha_{j}}^{-1}, \quad \eta_{i j}=t_{\beta_{i}} \circ t_{\beta_{j}} \circ t_{\beta_{i}+\beta_{j}}^{-1}, \\
v_{i j}=t_{\alpha_{i}} \circ t_{\beta_{j}} \circ t_{\alpha_{i}+\beta_{j}}^{-1} .
\end{gathered}
$$


Lemma 9.1. The following formulas hold true for $i \neq j=1, \ldots, g$ :

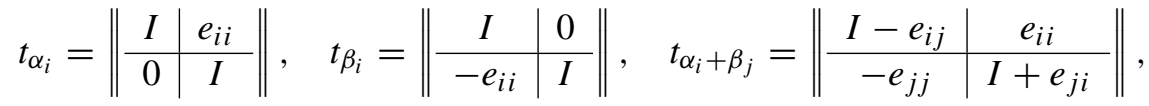

$$
\begin{aligned}
& t_{\alpha_{i}+\alpha_{j}}=\| \begin{array}{|l|l||}
I & e_{i i}+e_{j j}+e_{i j}+e_{j i} \\
\hline 0 & I
\end{array}, \\
& t_{\beta_{i}+\beta_{j}}=\| \begin{array}{c|c}
I & 0 \\
\hline-e_{i i}-e_{j j}-e_{i j}-e_{j i} & I
\end{array},
\end{aligned}
$$

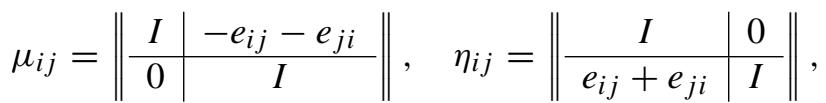

$$
\begin{aligned}
& v_{i j}=\left\|\begin{array}{c|c}
I+e_{i j} & 0 \\
\hline 0 & I-e_{j i}
\end{array}\right\| \text {. }
\end{aligned}
$$

Moreover, the matrices $t_{\alpha_{i}}, t_{\beta_{i}}, \mu_{i j}, \eta_{i j}$, and $v_{i j}(i \neq j=1, \ldots, g)$ generate $\operatorname{Sp}_{2 g}(\mathbb{Z})$.

Proof. The lemma can be established by direct calculations. The fact that these matrices generate $\mathrm{Sp}_{2 g}(\mathbb{Z})$ can be easily deduced from [OM, Ch. 2, §2.2.] or [B3].

For each $x \in \mathbb{Z}^{2 g}$ denote by $T(x)$ the subgroup in $\operatorname{Sp}_{2 g}(\mathbb{Z})$ generated by transvections along elements of $\mathbb{Z}^{2 g}$ that are $\omega$-orthogonal to $x$, i.e.

$$
T(x)=\left\langle t_{\gamma} \mid \gamma \in \mathbb{Z}^{2 g}, \omega(\gamma, x)=0\right\rangle .
$$

Also, let $\operatorname{St}(x)$ be the stabilizer of $x$ in $\mathrm{Sp}_{2 g}(\mathbb{Z})$, i.e.

$$
\operatorname{St}(x)=\left\{h \in \operatorname{Sp}_{2 g}(\mathbb{Z}) \mid h(x)=x\right\} .
$$

It easily follows from (9.3) that $T(x) \subset \mathrm{St}(x)$.

Proposition 9.2. $T\left(\alpha_{1}\right)=\operatorname{St}\left(\alpha_{1}\right)$. Moreover, this group is generated by the following matrices:

$$
t_{\alpha_{i}}, \quad t_{\beta_{i}}, \quad \mu_{i j}, \quad \eta_{i j}, \quad v_{i j}
$$

except for $t_{\beta_{1}}, \eta_{1 i}=\eta_{i 1}$ and $v_{i 1}(i \neq j=1, \ldots, g)$.

Proof. Evidently, the matrices (9.6) belong to $T\left(\alpha_{1}\right)$. Let $h \in \operatorname{St}\left(\alpha_{1}\right)$. We will show that $h$ is generated by (9.6). The proof consists of two steps.

Step 1. We will find an element $h_{1} \in \mathrm{Sp}_{2 g}(\mathbb{Z})$ such that $h \cdot h_{1}^{-1}$ is generated by (9.6) and $h_{1}\left(\beta_{1}\right)=\beta_{1}$. Let

$$
h\left(\beta_{1}\right)=a_{1} \alpha_{1}+b_{1} \beta_{1}+a_{2} \alpha_{2}+b_{2} \beta_{2}+\cdots,
$$


for some $a_{i}, b_{i} \in \mathbb{Z},(i=1, \ldots, g)$. Since $h$ preserves the form $\omega$ and $h\left(\alpha_{1}\right)=\alpha_{1}$, we get

$$
b_{1}=\omega\left(\alpha_{1}, h\left(\beta_{1}\right)\right)=\omega\left(h\left(\alpha_{1}\right), h\left(\beta_{1}\right)\right)=\omega\left(\alpha_{1}, \beta_{1}\right)=1 .
$$

Consider now the effect of action of $\mu_{1 j}$ and $v_{1 j}$ on $h\left(\beta_{1}\right), j=2, \ldots, g$. Let $t \in \mathbb{Z}$. Then it is easy to verify that for $j>1$ we have:

$$
\begin{aligned}
& \left(\mu_{1 j}\right)^{t} \circ h\left(\beta_{1}\right)=\left(a_{1}-t b_{j}\right) \alpha_{1}+\beta_{1}+\cdots+\left(a_{j}-t\right) \alpha_{j}+b_{j} \beta_{j}+\cdots, \\
& \left(v_{1 j}\right)^{t} \circ h\left(\beta_{1}\right)=\left(a_{1}+t a_{j}\right) \alpha_{1}+\beta_{1}+\cdots+a_{j} \alpha_{j}+\left(b_{j}-t\right) \beta_{j}+\cdots,
\end{aligned}
$$

where the coefficients at other basis elements are not changed.

Define now $h_{1} \in \mathbb{Z}^{2 g}$ by the formula

$$
h_{1}=\left(t_{\alpha_{1}}\right)^{-a^{\prime}} \cdot \prod_{j=2}^{g}\left(v_{1 j}\right)^{b_{j}} \cdot \prod_{i=2}^{g}\left(\mu_{1 j}\right)^{a_{j}} \cdot h,
$$

where

$$
a^{\prime}=a_{1}-\sum_{j=2}^{g} a_{j} b_{j}
$$

We claim that $h_{1}\left(\beta_{1}\right)=\beta_{1}$.

Indeed, the product of $\mu_{1 j}$ reduces the coefficients at $\alpha_{j}$ and the product of $v_{1 j}$ reduces the coefficients at $\beta_{j}$ for every $j=2, \ldots, g$. This also makes the coefficient at $\alpha_{1}$ equal to $a^{\prime}$. Since

$$
t_{\alpha_{1}}\left(\alpha_{1}\right)=\alpha_{1} \quad \text { and } \quad\left(t_{\alpha_{1}}\right)^{t}\left(\beta_{1}\right)=\left(a_{1}+t\right) \alpha_{1}+\beta_{1},
$$

we obtain that the multiple $\left(t_{\alpha_{1}}\right)^{-a^{\prime}}$ reduces this coefficient.

Step 2. Consider the following submodules of $\mathbb{Z}^{2 g}$ :

$$
P=\left\langle\alpha_{1}, \beta_{1}\right\rangle \text { and } Q=\left\langle\alpha_{i}, \beta_{i} \mid i=2, \ldots, g\right\rangle .
$$

They are orthogonal with respect to the form $\omega$ and $\left.h_{1}\right|_{P}=$ id. Since $h_{1}$ preserves $\omega$, it follows that $h_{1}(Q)=Q$. Thus $h_{1}$ can be regarded as an element of the group $\mathrm{Sp}_{2 g-2}(\mathbb{Z}) \subset \mathrm{Sp}_{2 g}(\mathbb{Z})$ consisting of isomorphisms that are the identity on $P$.

By Lemma 9.1 the group $\operatorname{Sp}_{2 g-2}(\mathbb{Z})$ is generated by matrices $(9.6)$ for $i \neq j=$ $2, \ldots, g$. In particular, they generate $h_{1}$.

\section{Minimal Morse maps}

For the proof of Theorem 8.5 we need the notion of minimal Morse mappings. Let $M$ be a compact surface, orientable or not. We say that a Morse map $f: M \rightarrow P$ 
is minimal if the number $c_{0}(f)+c_{1}(f)+c_{2}(f)$ of critical points of $f$ is minimal among all possible Morse maps $M \rightarrow P$ having the same sets of positive and negative boundary components as $f$. Let $b_{+}$and $b_{-}$be the number of $f$-positive and $f$ negative boundary components of $M$. The following lemma is easy to prove:

Lemma 10.1. A Morse mapping $f: M \rightarrow P$ is minimal if and only if for every connected component $X$ of $M$ the restriction $\left.f\right|_{X}$ is minimal. A Morse function $f: M \rightarrow \mathbb{R}^{1}$ on a connected surface $M$ is minimal if and only if the following two relations hold true:

$$
c_{0}(f)=\left\{\begin{array}{ll}
1, & \text { if } b_{-}=0, \\
0, & \text { if } b_{-}>0,
\end{array} \quad c_{2}(f)= \begin{cases}1, & \text { if } b_{+}=0 \\
0, & \text { if } b_{+}>0 .\end{cases}\right.
$$

Let $f: M \rightarrow S^{1}$ be a Morse mapping which is not null-homotopic. Then $f$ is minimal if and only if $c_{0}(f)=c_{2}(f)=0$.

We admit now that $M$ may be not connected. Let $f: M \rightarrow[0,1]$ be a Morse function such that $\frac{1}{2} \in[0,1]$ is its regular value. Denote

$$
\begin{gathered}
V_{0}=f^{-1}[0,1 / 2], \quad V_{1}=f^{-1}[1 / 2,1] . \\
B_{0}=f^{-1}(0), \quad B_{1}=f^{-1}(1), \quad Z=f^{-1}(1 / 2) .
\end{gathered}
$$

Lemma 10.2. Suppose that

(1) $B_{0}, B_{1}$ and $Z$ are nonempty, the union $B_{0} \cup B_{1}$ is included in $\partial M$ and intersects every connected component of $M$ non trivially;

(2) the restriction $\left.f\right|_{V_{i}}$ is a minimal Morse function for $i=0,1$;

(3) for every connected component $X$ of $M$ such that $X \cap Z \neq \varnothing$ we have $X \cap B_{i} \neq \varnothing$ for both $i=0,1$.

Then $f$ is a minimal Morse function on $M$.

Proof. Let $X$ be a component of $M$. We will show that $\left.f\right|_{X}$ is a minimal Morse function. Denote $X_{i}=X \cap V_{i}(i=0,1)$.

If $X \cap Z=\varnothing$, then $X$ is a connected component of either one of the sets $V_{0}$ or $V_{1}$. Then the restriction of $f$ onto $X$ is minimal.

Suppose that $X \cap Z \neq \varnothing$. Then $X \cap B_{i} \neq \varnothing$ for $i=0,1$ by (3). Evidently, the components of the intersection $X \cap Z \neq \varnothing$ are negative for the restriction $\left.f\right|_{X_{1}}$ and positive for the restriction $\left.f\right|_{X_{0}}$. Therefore, by Lemma 10.1, we have

$$
c_{2}\left(\left.f\right|_{X_{0}}\right)=c_{0}\left(\left.f\right|_{X_{1}}\right)=0 .
$$


Similarly, the intersection $X \cap B_{0}$ (resp. $X \cap B_{1}$ ) consists of some negative (resp. positive) components of $\left.f\right|_{X}$ and $\left.f\right|_{X_{0}}$ (resp. $\left.f\right|_{X_{1}}$ ). Then from Lemma 10.1, we also get

$$
c_{0}\left(\left.f\right|_{X_{0}}\right)=c_{2}\left(\left.f\right|_{X_{1}}\right)=0 .
$$

Combining this with (10.2), we obtain

$$
c_{i}\left(\left.f\right|_{X}\right)=c_{i}\left(\left.f\right|_{X_{0}}\right)+c_{i}\left(\left.f\right|_{X_{1}}\right)=0, \quad i=0,2 .
$$

Hence by Lemma $\left.10.1 f\right|_{X}$ is minimal.

\section{Minimization of intersections with a level-set}

Let $M$ be a compact surface (orientable or not), let $f: M \rightarrow S^{1}$ be a Morse mapping, and let $\gamma_{1}, \ldots, \gamma_{m} \subset M$ be disjoint SCCs.

Lemma 11.1. $f$ is $\Sigma$-homotopic to a Morse mapping $g$ such that for some level-set $L$ of $g$ and for every $i=1, \ldots, m$ the curve $\gamma_{i}$ does not pass through the critical points of $g$ and

(i) if the restriction $\left.f\right|_{\gamma_{i}}$ is not null-homotopic, then $\gamma_{i}$ transversely intersects every level-set of $g$;

(ii) otherwise $\gamma_{i} \cap L=\varnothing$.

Proof. Let $c \in S^{1}$ be a regular value of $f$. Set

$$
\Gamma=\bigcup_{i=1}^{m} \gamma_{i}, \quad n=\#\left[f^{-1}(c) \cap \Gamma\right], \quad \text { and } \quad d=\sum_{i=1}^{m}|\operatorname{deg} f| \gamma_{i} \mid .
$$

Then $\#\left[f^{-1}(c) \cap \gamma_{i}\right] \geq\left.\operatorname{deg} f\right|_{\gamma_{i}}$ for $i=0$, whence $n \geq d$. Moreover, $n=d$ if and only if $\#\left[f^{-1}(c) \cap \gamma_{i}\right]=\left.\operatorname{deg} f\right|_{\gamma_{i}}$.

Claim 11.2. Suppose that $n>d$. Then $f$ is $\Sigma$-homotopic to a Morse map $f_{1}$ such that $\#\left[f_{1}^{-1}\left(c_{1}\right) \cap \Gamma\right]<n$ for some regular value $c_{1}$ of $f_{1}$.

Proof. We will exploit the notations and the construction of Section 3. Cutting $M$ along $f^{-1}(c)$ we obtain the surface $\widetilde{M}$ and the Morse function $\widetilde{f}: \widetilde{M} \rightarrow[0,1]$. Let also $p: \widetilde{M} \rightarrow M$ be the factor-map, $B_{i}=\widetilde{f}^{-1}(i)$ for $i=0$, 1 , and $B=B_{0} \cup B_{1}=$ $p^{-1}\left(f^{-1}(c)\right)$.

Let $L=p^{-1}(\Gamma)$ and let $l_{1}, \ldots, l_{k}$ be the connected components of $L$. Then the intersection $l_{j} \cap B$ is either empty (whence $l_{j}$ is an SCC) or consists of two points (whence $l_{j}$ is a simple arc with ends in $B$ ). Let us divide $L$ into four groups 
$L_{\varnothing}, L_{0}, L_{0}^{1}, L^{1}$ consisting of arcs that respectively do not intersect $B$, have nonempty intersections only with $B_{0}$, with both sets $B_{1}$ and $B_{0}$, and with $B_{1}$ only. Thus

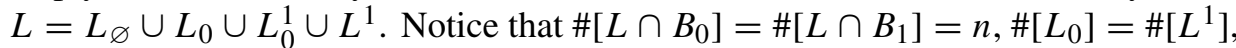
and the sets $L_{0}$ and $L^{1}$ are non-empty if and only if $n>d$.

Let $Q_{0}^{1} \subset \widetilde{M}$ be the union of those connected components of $\tilde{M}$ which intersect both sets $B_{0}$ and $B_{1}$ non trivially. Consider the set

$$
G=Q_{0}^{1} \cap\left(B_{0} \cup L_{0}\right) .
$$

By definition, $G \cap\left(L_{\varnothing} \cup L^{1}\right)=\varnothing$. Then there exists a regular neighborhood $W$ of $G$ which does not intersect $L_{\varnothing} \cup L^{1}$ and such that the boundary $Z=\partial W$ transversely intersects every component of $L_{0}^{1}$ at a unique point. Hence, $Z \cap L=Z \cap L_{0}^{1}$. Evidently, $Z$ separates $\tilde{M}$ between $B_{0}$ and $B_{1}$. Moreover, $\#\left[Z \cap L_{0}^{1}\right]<n$.

We will now construct a Morse function $\widetilde{g}: \widetilde{M} \rightarrow[0,1]$ which coincides with $\tilde{f}$ in some neighborhood of $B \cup \partial \tilde{M}$, has the critical type of $\tilde{f}$, and such that $\tilde{g}^{-1}\left(\frac{1}{2}\right)=Z$.

Let $\widetilde{g}_{0}: V_{0} \rightarrow\left[0, \frac{1}{2}\right]$ and $\widetilde{g}_{1}: V_{1} \rightarrow\left[\frac{1}{2}, 1\right]$ be two minimal Morse functions such that

$$
\tilde{g}_{0}^{-1}(0)=B_{0}, \quad \tilde{g}_{0}^{-1}(1 / 2)=\widetilde{g}_{1}^{-1}(1 / 2)=Z, \quad \tilde{g}_{1}^{-1}(1)=B_{1},
$$

and the Morse function $\widetilde{g}: \widetilde{M} \rightarrow[0,1]$ defined by $\left.\widetilde{g}\right|_{V_{i}}=\widetilde{g}_{i}(i=0,1)$ is $C^{\infty}$, has the same sets of positive and negative components as $\widetilde{f}$, and coincides with $\tilde{f}$ in some neighborhood of $B \cup \partial \widetilde{M}$.

We claim that $\tilde{g}$ is minimal. Indeed, let $X$ be a component of $\tilde{M}$ such that $X \cap Z \neq \varnothing$. Since $Z=\partial W \subset Q_{0}^{1}$, we obtain that $X \subset Q_{0}^{1}$. Denote $X_{i}=X \cap V_{i}$, then $X \cap B_{i}=X_{i} \cap B_{i} \neq \varnothing$, by the definition of $Q_{0}^{1}$. It follows from Lemma 10.2 that $\tilde{g}$ is minimal.

Adding critical points to $\tilde{g}$ outside of $B \cup Z$ we can change its critical type to the critical type of $\widetilde{f}$. Let us denote this new function by $\widetilde{f}_{1}$. Then $\widetilde{f}_{1}$ satisfies the statement of our claim.

Indeed, denote $c_{1}=q\left(\frac{1}{2}\right)$. By the case $P=\mathbb{R}^{1}$ of the Main Theorem we obtain that $\tilde{f} \stackrel{\Sigma}{\sim} \tilde{f}_{1}$ with respect to some neighborhood of $B \cup \partial \tilde{M}$. This $\Sigma$-homotopy induces a $\Sigma$-homotopy (with respect to $f^{-1}(c) \cup \partial M$ ) of $f$ to a Morse mapping $f_{1}$ such that $\#\left[f_{1}^{-1}\left(c_{1}\right) \cap \Gamma\right]<n$.

We now proceed with the proof of Lemma 11.1. By Claim 11.2 we can assume that $n=d$. As noted above this is equivalent to the statement $\#\left[f^{-1}(c) \cap \gamma_{i}\right]=\left.\operatorname{deg} f\right|_{\gamma_{i}}$. In particular, if the restriction $\left.f\right|_{\gamma_{i}}$ is null-homotopic, then $\#\left[f^{-1}(c) \cap \gamma_{i}\right]=0$, i.e. $\gamma_{i} \cap f^{-1}(c)=\varnothing$, whence (ii) holds true.

Let us assume that $l_{i}$ is given by an embedding $l_{i}:[0,1] \rightarrow \tilde{M}$ so that $l_{i} \cap l_{j}=\varnothing$ for $j \neq i$. To establish (i) we prove that following claim: 
Claim 11.3. Suppose that $l_{i}(0) \in B_{0}, l_{i}(1) \in B_{1}$, and that the intersection $l_{i} \cap B$ is transversal for each $i=1, \ldots, k$. Then $\widetilde{f}$ is $\Sigma$-homotopic to a Morse function $\widetilde{g}$ such that $l_{i}$ is transversal to level-sets of $\widetilde{g}$.

It follows that a $\Sigma$-homotopy of this claim yields a $\Sigma$-homotopy $f \stackrel{\Sigma}{\sim} g$ with respect to $f^{-1}(c)$ such that every $\gamma_{i}$ is transversal to level-sets of $g$. This will complete Lemma 11.1.

Proof of Claim 11.3. We will construct a Morse function $\widetilde{f}_{1}$ and a gradient-like vector field $F$ for $\tilde{f}_{1}$ such that for every $i=1, \ldots, m$ the arc $l_{i}$ is a trajectory of $F$. Then adding or canceling the proper number of pairs of critical points of $\widetilde{f_{1}}$ outside of $\bigcup_{i} l_{i}$ we obtain a Morse function $\widetilde{g}$ having the critical type of $\widetilde{f}$ and such that $F$ is a gradient-like for $\tilde{g}$.

For every $i=1, \ldots, m$ let $\phi_{i}:[0,1] \times[-1,1] \rightarrow M$ be a smooth embedding such that the image $V_{i}=\operatorname{Im} \phi_{i}$ is a neighborhood of $l_{i}, \phi_{i}(t, 0)=l_{i}(t)$ for $t \in[0,1]$, $\phi^{-1}\left(B_{S}\right)=\{s\} \times[-1,1]$ for $s=0,1$. Since the $l_{i}$ are mutually disjoint, we can assume that so are the $V_{i}$. Denote $V=\bigcup_{i=1}^{m} V_{i}$ and define a function $\tilde{g}: V \rightarrow[0,1]$ by the formula $\tilde{g}(x)=p_{2} \circ \phi_{i}^{-1}(x)$ for $x \in V_{i}$, where $p_{2}:[0,1] \times[-1,1] \rightarrow[-1,1]$ is the natural projection.

Slightly changing $\widetilde{g}$ outside some neighborhood of $\bigcup_{i} l_{i}$ we can extend $\widetilde{g}$ over all of $\widetilde{M}$. Moreover, this extension may be assumed Morse whose positive and negative boundary components coincide with the ones of $\tilde{f}$ though the number of critical points of $\tilde{g}$ and $\tilde{f}$ may be different. Now we show how to change the critical type $K(\tilde{f})$ of $\widetilde{g}$ by adding or canceling pairs of critical points outside of $\bigcup_{i} l_{i}$.

Recall that a vector field $F$ on a manifold $\widetilde{M}$ is gradient-like for a function $\tilde{f}: \widetilde{M} \rightarrow \mathbb{R}^{1}$ if $d \widetilde{f}(F)(x)>0$ at each regular point $x$ of $\widetilde{f}$.

Let $\Psi$ be any gradient-like vector field for the function $\widetilde{g}$ on $\tilde{M}$ and let $\widetilde{\Phi}$ be the gradient vector field for the function $p_{2}$ on $[0,1] \times[-1,1]$, i.e. $\widetilde{\Phi}(s, t)=(0,1)$. Using $\phi_{i}$ we transfer $\widetilde{\Phi}$ to $V_{i}$. This gives us a vector field $\Phi$ on $V$ such that $l_{i}$ is a trajectory of $\Phi$ for $i=1, \ldots, m$.

Finally, we glue $\Psi$ and $\Phi$. Let $V^{\prime}$ be a neighborhood of $\bigcup_{i} l_{i}$ such that $\overline{V^{\prime}} \subset V$ and let $W=\tilde{M} \backslash \overline{V^{\prime}}$. Then $V \cup W=\tilde{M}$.

Let $\mu_{1}, \mu_{2}: \widetilde{M} \rightarrow[0,1]$ be a partition of unity corresponding to the open covering $\{V, W\}$ of $\widetilde{M}$, i.e. supp $\mu_{1} \subset V$, supp $\mu_{2} \subset W$, and $\mu_{1}+\mu_{2} \equiv 1$. Define a vector field $F$ on $\tilde{M}$ by the formula

$$
F(x)=\mu_{1}(x) \cdot \Phi(x)+\mu_{2}(x) \cdot \Psi(x), \quad x \in \tilde{M} .
$$

Evidently, $F$ is gradient-like for $\tilde{g}$ and coincides with $\Phi$ near $\bigcup_{i} l_{i}$. In particular, every $l_{i}$ is a trajectory of $F$, whence $l_{i}$ transversely intersects level-sets of $\widetilde{g}$.

It remains to show that $\tilde{g}$ can be changed outside of $\bigcup_{i} l_{i}$ to have the critical type of $\tilde{f}$. First we show how to make $\widetilde{g}$ a minimal Morse function. 
Suppose that $\tilde{g}$ has a critical point $z_{0}$ either of index 0 or 2 . Since the sets of positive and negative boundary components of $\widetilde{g}$ are non-empty, there exists a critical points $z_{1}$ of index 1 and a trajectory $\omega$ of $F$ with ends at $z_{0}$ and $z_{1}$. This trajectory does not intersect $\bigcup l_{i}$. Hence $\widetilde{g}$ can be changed in some neighborhood $N$ of $\omega$ to have no critical points in $N$ (see [HM], [MJ1]). Thus the number of critical points is reduced. By a similar procedure we can add pairs of critical points outside of $\bigcup_{i} l_{i}$. Therefore we can change the critical type $K(\widetilde{f})$ of $\widetilde{g}$ leaving $l_{i}$ transversal to level-sets of $\widetilde{g}$.

\section{Proof of (i) of Theorem 8.5}

Let $\gamma \subset M$ be a simple closed curve and let $t_{\gamma}$ be a Dehn twist along $\gamma$.

Necessity. Suppose that $t_{\gamma}$ is $f$-admissible. Then $f$ and $f \circ t_{\gamma}$ are homotopic. We should show that $\left.\operatorname{deg} f\right|_{\gamma}=0$. We can assume that there is a regular value $c$ of $f$ such that $\alpha=f^{-1}(c)$ is an SCC. Denote $\alpha^{\prime}=t_{\gamma}(\alpha)$.

Since $f$ and $f \circ t_{\gamma}$ are homotopic, we obtain from the last paragraph of Section 3.1 that $\left[\alpha^{\prime}\right]=[\alpha]$ in $H_{1}(M, \partial M)$, i.e. $t_{\gamma}$ fixes $[\alpha]$. Then by Eq. (9.3) for the action of Dehn twists in $H_{1}(M, \partial M)$ we get

$$
[\alpha]=t_{\gamma}([\alpha])=\omega([\gamma],[\alpha]) \cdot[\gamma]+[\alpha]=\left.\operatorname{deg} f\right|_{\gamma} \cdot[\gamma]+[\alpha],
$$

whence $\left.\operatorname{deg} f\right|_{\gamma}=0$.

Sufficiency. Suppose that $\left.f\right|_{\gamma}$ is null-homotopic. By Lemma 11.1, $f$ is $\Sigma$ homotopic to a Morse mapping $g$ such that $g^{-1}(c) \cap \gamma=\varnothing$ for some regular value $c$ of $g$. We now apply the construction of Section 3. Cutting $M$ along $g^{-1}(c)$ we obtain a surface $\widetilde{M}=\widetilde{M}(g, c)$, a Morse function $\widetilde{g}: \widetilde{M} \rightarrow[0,1]$, and an SCC $\widetilde{\gamma} \subset \widetilde{M}$ corresponding to $\gamma$. From the case $P=\mathbb{R}^{1}$ of the Main Theorem, $t \widetilde{\gamma}$ is $\widetilde{g}$-admissible. Then $t_{\gamma}$ is $g$-admissible and therefore $f$-admissible.

\section{Proof of (ii) of Theorem 8.5}

Let $f: M \rightarrow S^{1}$ be a Morse mapping, let $\gamma_{1}, \gamma_{2}$ be disjoint oriented homologous simple closed curves in $M$, and let $t=t_{\gamma_{1}} \circ t_{\gamma_{2}}^{-1}$ be the product of Dehn twists along these curves. We must prove that $t$ is $f$-admissible.

Since these curves are homologous, it follows that the restrictions of $f$ to them are homotopic. If these restrictions are null-homotopic, then by the case (i) of this theorem $t$ is $f$-admissible. Therefore we will assume that $\left.f\right|_{\gamma_{1}} \nsim 0$.

By Lemma 11.1 we can also assume that $\gamma_{i}$ transversely intersects each level-set of $g$. Then the statement (ii) of Theorem 8.5 is a direct corollary of the following lemma: 
Lemma 13.1. Let $f: M \rightarrow S^{1}$ be a Morse mapping, let $\gamma_{1}, \gamma_{2}$ be two disjoint homologous SCCs in $M$, and let $t=t_{\gamma_{1}} \circ t_{\gamma_{2}}^{-1}$. Suppose that both of the $\gamma_{i}$ transversely intersect every level-set of $f$. Then $f \stackrel{\Sigma}{\sim} f \circ t$.

Proof. Let $X \subset M$ be the closure of one of the connected components of $M \backslash\left(\gamma_{1} \cup \gamma_{2}\right)$ bounded by the curves $\gamma_{1}$ and $\gamma_{2}$. Since $\gamma_{k}(k=1,2)$ transversely intersects levelsets of $g$, there exists an embedding $\phi_{k}$ of $S^{1} \times[-2,2]$ onto some neighborhood $N_{k}$ of $\gamma_{k}$ such that

$$
\phi_{k}\left(S^{1} \times\{0\}\right)=\gamma_{k}, \quad \phi_{k}\left(S^{1} \times[0,2]\right) \subset X,
$$

and the following diagram is commutative:

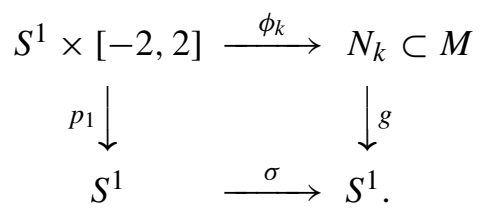

Here $p_{1}$ is a projection onto the first coordinate and $\sigma$ is a covering mapping of degree $d=\left.\operatorname{deg} f\right|_{\gamma_{1}}=\left.\operatorname{deg} f\right|_{\gamma_{2}}$ defined by the formula $\sigma(z)=z^{d}$. Thus

$$
g \circ \phi_{k}(z, t)=z^{d} .
$$

We can also assume that $N_{1} \cap N_{2}=\varnothing$. To simplify notation, for each pair $a, b \in$ $[-2,2]$ we denote

$$
N_{k}^{[a, b]}=\phi_{k}\left(S^{1} \times[a, b]\right) .
$$

Let $\mu:[-2,2] \rightarrow[0,1]$ be a $C^{\infty}$ function such that $\mu[-2,-1]=0$ and $\mu[1,2]=1$. Then the Dehn twist $t_{\gamma_{k}}$ along $\gamma_{k}$ can be defined so that $t=t_{\gamma_{1}} \circ t_{\gamma_{2}}^{-1}$ will have the form

$$
t(z, t)= \begin{cases}x, & x \in M \backslash\left(N_{1} \cup N_{2}\right), \\ \left(z e^{2 \pi i \mu(s)}, s\right), & x=\phi_{k}(z, s) \in N_{k}, k=1,2 .\end{cases}
$$

Now a $\Sigma$-homotopy $G: M \times[0,1] \rightarrow S^{1}$ between $g$ and $g \circ t$ can be defined by the formula

$$
G(x, t)= \begin{cases}g(x) e^{2 \pi i d t}, & x \in X \backslash\left(N_{1}^{[0,1]} \cup N_{2}^{[0,1]}\right), \\ g \circ \phi_{k}\left(z e^{2 \pi i \mu(s) \cdot t}, s\right), & x=\phi_{k}(z, s) \in N_{k}, k=1,2, \\ g(x), & x \in M \backslash\left(X \cup N_{1}^{[-1,0]} \cup N_{2}^{[-1,0]}\right) .\end{cases}
$$

Remark 13.2. A geometrical meaning of this formula is that the mapping $G$ "moves" $d$ times the part $X$ between the curves $\gamma_{1}$ and $\gamma_{2}$ "around $S^{1}$ " leaving the the complement $M \backslash X$ fixed. 
Let us verify, that $G$ is in fact a $\Sigma$-homotopy connecting $g$ with $g \circ t$.

Proof. It is clear that $G_{0}=g$. Moreover, it follows from (13.1) and (13.2) that $\phi_{1}$ preserves orientation of $S^{1} \times[-2,2]$ while $\phi_{2}$ reverses it. Hence by (13.4) we get $G_{1}=g \circ t_{\gamma_{1}} \circ t_{\gamma_{2}}^{-1}$.

Evidently, the continuity of $G$ will imply its smoothness. To prove that $G$ is continuous we should verify that the second formula coincides with the first one on $N_{1}^{[1,2]} \cup N_{2}^{[1,2]}$ and with the third one on $N_{1}^{[-2,-1]} \cup N_{2}^{[-2,-1]}$.

Let $x=\phi_{k}(z, s) \in N_{k}^{[1,2]}$ for $k=1,2$, then $\mu(s)=1$, whence, using (13.3), we get

$$
g \circ \phi_{k}\left(z e^{2 \pi i \mu(s) \cdot t}, s\right)=z^{d} e^{2 \pi i d t}=g(x) e^{2 \pi i d t} .
$$

Let now $x=\phi_{k}(z, s) \in N_{k}^{[-2,-1]}$ for $k=1,2$, then $\mu(s)=0$, whence

$$
g \circ \phi_{i}\left(z e^{2 \pi i \mu(s) \cdot t}, s\right)=g \circ \phi_{i}(z, s)=g(x) .
$$

Notice that for every point $x \in M$ there exists a neighborhood on which $G_{t}$ differs from $g$ by a diffeomorphism of either $S^{1}$ or $M$. Hence $G_{t}$ is Morse for all $t \in[0,1]$, i.e. $G$ is a $\Sigma$-homotopy.

\section{Proof of Lemma 8.4}

Suppose that $h \in \mathcal{P} \mathcal{M}(M)$ is generated by $\left\{t_{l}: l \in \mathcal{C}\right\}$ and such that the mappings $f$ and $f \circ h$ are homotopic. We have to prove that $h$ is in fact generated by $\left\{t_{l}: l \in \mathcal{C} \backslash \beta_{1}\right\}$.

Recall that $H_{1}(M, \partial M)$ is a free module generated by homology classes of $\alpha_{1}, \ldots, \alpha_{g}, \beta_{1}, \ldots, \beta_{g}$. Moreover, the matrix of $\omega$ in this basis has the form (9.2). Since $h_{*}$ preserves this $\omega$ we may suppose that $h_{*} \in \operatorname{Sp}_{2 g}(\mathbb{Z})$.

Notice that $h_{*}\left[\alpha_{1}\right]=\left[\alpha_{1}\right]$, since $\alpha_{1}$ is a level-set of $f$, whence $h_{*}$ belongs to the stabilizer $\operatorname{St}\left(\left[\alpha_{1}\right]\right)$ of $\alpha_{1}$ in $\operatorname{Sp}_{2 g}(\mathbb{Z})$.

Let $t_{\gamma}$ be a Dehn twist along a simple closed curve $\gamma$. Then it acts on $H_{1}(M, \partial M)$ by the following formula:

$$
\left(t_{\gamma}\right)_{*}(x)=\omega([\gamma], x) \cdot[\gamma]+x \quad \text { for all } x \in H_{1}(M),
$$

thus it is a transvection along $[\gamma]$, see Eq. (9.3).

Hence the products of transvections $\mu_{i j}, \eta_{i j}, v_{i j}$ defined by Formula (9.4) can be realized by products of Dehn twists. It follows from Theorem 8.5 that all these diffeomorphisms except for $\eta_{1 i}=\eta_{i 1}$ and $v_{i 1}$ are $f$-admissible.

On the other hand, by Proposition 9.2, $h_{*}$ is generated by the linear isomorphisms $t_{\alpha_{i}}, t_{\beta_{i}}, \mu_{i j}, \eta_{i j}, v_{i j}$, except for $t_{\beta_{1}} \eta_{1 i}=\eta_{i 1}$ and $v_{i 1}$, where $i \neq j=1, \ldots, g$.

Hence, there exists an $f$-admissible diffeomorphism $c$ of $M$ which induces the same isomorphism of $H$ as $h_{*}$. Then $t=c^{-1} \circ h$ belongs to $\mathcal{T}(M)$. 


\section{Appendix. Proof of the Main Theorem. Case $P=S^{1}$}

We extend here our proof of the Main Theorem given in [M] to the case when $M$ is arbitrary and $P=S^{1}$.

Let $f, g: M \rightarrow S^{1}$ be two Morse mappings of the same critical type, let $c$ be their common regular value, $\alpha=f^{-1}(c)$, and let $\gamma=g^{-1}(c)$. By Lemma 5.1 we can assume that the homomorphism $f_{*}=g_{*}: H_{1}(M) \rightarrow H_{1}\left(S^{1}\right)$ is onto and by Lemma 3.2 that $\alpha$ and $\gamma$ are connected, i.e. SCCs.

Let us cut $M$ along $\alpha$ and denote the obtained surface by $\tilde{M}$. Let also $p: \widetilde{M} \rightarrow M$ be the factor-mapping, $\widetilde{f}: \widetilde{M} \rightarrow[0,1]$ the corresponding Morse function induced by $f, B_{0}=\widetilde{f}^{-1}(0), B_{1}=\widetilde{f}^{-1}(1)$, and $B=B_{0} \cup B_{1}$ (we use the notations of Section 3).

Claim 14.1. If $\alpha=\gamma$, then $f \stackrel{\Sigma}{\sim} g$.

Proof. Since $f$ and $g$ are homotopic, we can assume (by small $\Sigma$-homotopy) that they coincide near $\alpha$. Then $g$ also yields a Morse function $\widetilde{g}: M \rightarrow[0,1]$ which coincides near $B$ with $\tilde{f}$ and $K(\tilde{f})=K(\widetilde{g})$. By the $\mathbb{R}$-case of the Main Theorem $\tilde{f} \stackrel{\Sigma}{\sim} \widetilde{g}$ with respect to a neighborhood of $B$. Then this $\Sigma$-homotopy yields a $\Sigma$-homotopy between $f$ and $g$ with respect to a neighborhood of $\alpha$.

Suppose that $\alpha \neq \gamma$. Since $f$ and $g$ are homotopic, it follows that the restriction $\left.f\right|_{\gamma}$ is null-homotopic. Then by Lemma 11.1 we can additionally assume that $\alpha \cap \gamma=$ $\varnothing$.

In this case $\tilde{\gamma}=p^{-1}(\gamma)$ separates $\tilde{M}$ between $B_{0}$ and $B_{1}$. Using the method of Claim 11.2 we can construct a Morse function $\widetilde{f}_{1}: \widetilde{M} \rightarrow[0,1]$ which coincides with $\tilde{f}$ near $B_{0} \cup B_{1}$, has the critical type of $\tilde{f}$, and such that $f_{1}^{-1}\left(\frac{1}{2}\right)=\tilde{\gamma}$. Then $\widetilde{f}_{1}$ yields a Morse mapping $f_{1}: M \rightarrow S^{1}$ which coincides with $\tilde{f}$ in a neighborhood of $\alpha$ and such that $f_{1}^{-1}\left(p\left(\frac{1}{2}\right)\right)=\gamma$. Thus $\alpha$ and $\gamma$ are level-sets of $f_{1}$. Then by Claim 14.1 we get $f \stackrel{\Sigma}{\sim} f_{1} \stackrel{\Sigma}{\sim}$.

\section{Acknowledgments}

I am sincerely grateful to V. V. Sharko, A. Prishlyak, M. Pankov, E. Polulyakh, I. Vlasenko for many valuable discussions. I am deeply thankful to E. Kudryavtseva for the valuable discussions. I wish to thank the referee for pointing out to the nonorientable case of the Main Theorem, referring me to the paper [KA], and proposing the right name for Kronrod-Reeb graphs. I thank A. Pankov for the information about paper $[\mathrm{KM}]$. I also thank B. Szepietowski for sending me his paper [SB]. 
Vol. 80 (2005)

\section{References}

[B1] J. S. Birman, On braid groups. Comm. Pure Appl. Math. 22 (1969), 41-72. Zbl 0157.30904 MR 0234447

[B2] J. S. Birman, Mapping class groups and their relation to braid groups. Comm. Pure Appl. Math. 22 (1969), 213-238. Zbl 0167.21503 MR 0243519

[B3] J. S. Birman, On Siegel's modular group. Math. Ann. 191 (1971), 59-68. Zbl 0208.10601 MR 0280606

[BC] J. S. Birman, D. R. J. Chillingworth, On the homeotopy group of a non-orientable surface. Math. Proc. Cambridge Philos. Soc. 71 (1972), 437-448; Erratum, 136 (2004), 441. Zbl 0232.57001 MR 0300288 MR 2040584

[BF] A. V. Bolsinov, A. T. Fomenko, Introduction to the topology of integrable Hamiltonian systems. Nauka, Moscow 1997. Zbl 0908.58026 MR 1664068

[C] D. R. J. Chillingworth, A finite set of generators for the homeotopy group of a nonorientable surface. Proc. Cambridge Philos. Soc. 65 (1969), 409-430. Zbl 0172.48801 MR 0235583

[D] M. Dehn, Die Gruppe der Abbildungsklassen. (Das arithmetische Feld auf Flächen.) Acta Math. 69 (1938), 135-206. Zbl 0019.25301

[G] S. Gervais, A finite presentation of the mapping class group of a punctured surface. Topology 40 (2001), 703-725. Zbl 0992.57013 MR 1851559

[HT] A. Hatcher, W. Thurston, A presentation for the mapping class group of a closed orientable surface. Topology 19 (1980), 221-237. Zbl 0447.57005 MR 0579573

[H] M. W. Hirsch, Differential topology. Grad. Texts in Math. 33, Springer, New York 1976. Zbl 0356.57001 MR 1336822

[HH] M. D. Hirsch, M. W. Hirsch, Families of Morse functions parametrized by maxima. Michigan Math. J. 45 (1998), 347-368. Zbl 0968.57026 MR 1637670

[HM] W. Huebsch, M. Morse, The bowl theorem and a model nondegenerate function. Proc. Nat. Acad. Sci. U.S.A. 51 (1964), 49-51. Zbl 0124.05901 MR 0160245

[I] K. Igusa, On the homotopy type of the space of generalized Morse functions. Topology 23 (1984), 245-256. Zbl 0595.57025 MR 0744854

[IS] K. Ikegami, O. Saeki, Cobordism group of Morse functions on surfaces. J. Math. Soc. Japan 55 (2003), 1081-1094. Zbl 1046.57020 MR 2003761

[J] D. L. Johnson, Homeomorphisms of a surface which act trivially on homology. Proc. Amer. Math. Soc. 75 (1979), 119-125. Zbl 0407.57003 MR 0529227

[KM] M. Korkmaz, Mapping class groups of nonorientable surfaces. Geom. Dedicata 89 (2002), 109-133. Zbl 1016.57013 MR 1890954

[KA] A. S. Kronrod, On functions of two variables. Uspehi Mat. Nauk. 5 (1) (1950), 24-134 (in Russian). Zbl 0040.31603 MR 0034826

[KE] E. A. Kudryavtseva, Realization of smooth functions on surfaces as height functions. Mat. Sb. 190 (1999), 29-88 (in Russian). Zbl 0941.57026 MR 1700994

[K] E. V. Kulinich, On topologically equivalent Morse functions on surfaces. Methods Funct. Anal. Topology 4 (1) (1998), 59-64. Zbl 0934.57036 MR 1770821 
[L1] W. B. R. Lickorish, A finite set of generators for the homeotopy group of a 2-manifold. Proc. Cambridge Philos. Soc. 60 (1964), 769-778. Zbl 0131.20801 MR 0171269

[L2] W. B. R. Lickorish, Homeomorphisms of non-orientable two manifolds. Proc. Cambridge Philos. Soc. 59 (1963), 307-317. Zbl 0115.40801 MR 0145498

[L3] W. B. R. Lickorish, On the homeomorphisms of non-orientable surface. Proc. Cambridge Philos. Soc. 61 (1965), 61-64. Zbl 0131.20802 MR 0169221

[M] S. Maksymenko, Components of spaces of Morse mappings. In Some problems in contemporary mathematics, Pr. Inst. Mat. Nats. Akad. Nauk Ukr. Mat. Zastos. 25 (1998), 135-153 (in Russian). MR 1744357

[MG] G. Mess, The Torelli groups for genus 2 and 3 surfaces. Topology 31 (1992), 775-790. Zbl 0772.57025 MR 1191379

[MJ1] J. Milnor, Lectures on the h-coborsdism theorem. Notes by L. Siebenmann and J. Sondow, Princeton Math. Notes, Princeton University Press, Princeton, New Jersey, 1965. Zbl 0161.20302 MR 0190942

[MJ2] J. Milnor, Morse theory. Ann. of Math. Stud. 51, Princeton University Press, Princeton, New Jersey, 1963. Zbl 0108.10401 MR 0163331

[OM] O. T. O’Meara, Lectures on symplectic groups. University of Notre Dame, 1976.

[P] J. Powell, Two theorems on the mapping class group of a surface. Proc. Amer. Math. Soc. 68 (1978), 347-350. Zbl 0391.57009 MR 0494115

[SV1] V. V. Sharko, Functions on surfaces. I. In Some problems in contemporary mathematics, Pr. Inst. Mat. Nats. Akad. Nauk Ukr. Mat. Zastos. 25 (1998), 408-434 (in Russian). MR 1744373

[SV2] V. V. Sharko, Smooth and topological equivalence of functions on surfaces. Ukraïn. Mat. Zh. 55 (2003), 687-700; English transl. Ukrainian Math. J. 55 (2004), 253-260. Zbl 1039.58036 MR 2071708

[SB] B. Szepietowski, Involutions in mapping class groups of non-orientable surfaces. Collect. Math. 55 (3) (2004), 253-260. Zbl 02140883 MR 2099216

Received December 11, 2003; revised January 26, 2005

Sergey Maksymenko, Topology Department, Institute of Mathematics of Ukrainian NAS, Tereshchenkivska str. 3, 01601 Kyiv, Ukraine

E-mail: maks@imath.kiev.ua 